\title{
An efficient numerical method for forward-backward stochastic differential equations driven by $G$-Brownian motion *
}

\author{
Mingshang $\mathrm{Hu}^{\mathrm{a}, \mathrm{b}}$, Lianzi Jiang ${ }^{\mathrm{b}, *}$ \\ ${ }^{a}$ Zhongtai Securities Institute for Financial Studies, Shandong University, Jinan, Shandong 250100, PR \\ China \\ ${ }^{b}$ Zhongtai Securities Institute for Financial Studies, Shandong University, Jinan, Shandong 250100, PR \\ China
}

\begin{abstract}
In this paper, we study the numerical method for solving forward-backward stochastic differential equations driven by $G$-Brownian motion ( $G$-FBSDEs) which correspond to fully nonlinear partial differential equations (PDEs). First, we give an approximate conditional $G$-expectation and obtain some feasible methods to calculate the distribution of $G$-Brownian motion. On this basis, some efficient numerical schemes for $G$-FBSDEs are then proposed. We rigorously analyze the errors of the proposed schemes and prove the convergence. Finally, several numerical experiments are presented to demonstrate the accuracy of our schemes. Keywords: backward stochastic differential equations, $G$-Brownian motion, numerical schemes, fully nonlinear PDEs
\end{abstract}

\section{Introduction}

Considering the volatility uncertainty in the financial market, Avellaneda et al. [1], Lyons [23], and Dellacherie [8] initially studied the superhedging of European options. But for the superhedging of general path-dependence options, their methods are no longer suitable. 5 Recently, Peng $[26,27,28,29]$ introduced the $G$-expectation theory to deal with this problem, see also $[9,31]$ for different approaches. Under the $G$-expectation framework, a new notion of $G$-normal distribution was introduced, which is the limit distribution corresponding to the central limit theorem. The notion of $G$-normal distribution plays the same important role in the theory of sublinear expectation as that of normal distribution in the classical probability

\footnotetext{
* This work was funded by National Key R\&D Program of China (No. 2018YFA0703900) and the National Natural Science Foundation of China (No. 11671231).

*Corresponding author

Email addresses: humingshang@sdu.edu.cn (Mingshang Hu), jianglianzi95@163.com (Lianzi Jiang)
} 
theory. Based on it, a new type of $G$-Brownian motion and the related stochastic calculus of Itô's type have been established.

In this paper, we study the feasible numerical scheme for the following forward-backward stochastic differential equation driven by $G$-Brownian motion $(G$-FBSDE for short, we always use Einstein convention):

$$
\left\{\begin{aligned}
d X_{t} & =b\left(t, X_{t}\right) d t+h_{i j}\left(t, X_{t}\right) d\left\langle B^{i}, B^{j}\right\rangle_{t}+\sigma\left(t, X_{t}\right) d B_{t}, X_{0}=x_{0} \in \mathbb{R}^{m} \\
-d Y_{t} & =f\left(t, X_{t}, Y_{t}, Z_{t}\right) d t+g_{i j}\left(t, X_{t}, Y_{t}, Z_{t}\right) d\left\langle B^{i}, B^{j}\right\rangle_{t}-Z_{t} d B_{t}-d K_{t}, \\
Y_{T} & =\phi\left(X_{T}\right)
\end{aligned}\right.
$$

where $B_{t}=\left(B_{t}^{1}, \ldots, B_{t}^{d}\right)^{\top}$ is a $d$-dimensional $G$-Brownian motion defined in the $G$-expectation space $\left(\Omega_{T}, L_{G}^{1}\left(\Omega_{T}\right), \hat{\mathbb{E}}\right), K$ is a decreasing $G$-martingale, $b, h_{i j}:[0, T] \times \mathbb{R}^{m} \rightarrow \mathbb{R}^{m}, \sigma$ : $[0, T] \times \mathbb{R}^{m} \rightarrow \mathbb{R}^{m \times d}, \phi: \mathbb{R}^{m} \rightarrow \mathbb{R}, f, g_{i j}:[0, T] \times \mathbb{R}^{m} \times \mathbb{R} \times \mathbb{R}^{d} \rightarrow \mathbb{R}$, and $h_{i j}=h_{j i}$, $g_{i j}=g_{j i}$, for $1 \leq i, j \leq d$. The first equation in (1.1) is the stochastic differential equation driven by $G$-Brownian motion $(G$-SDE), and the second equation is the backward stochastic differential equation driven by $G$-Brownian motion ( $G$-BSDE).

Under some standard conditions on $b, h_{i j}, \sigma, f, g_{i j}$, and $\phi$, $\mathrm{Hu}$ et al. [19] proved the existence and uniqueness of the solution for $G$-BSDEs. Moreover, they [20] studied the nonlinear Feynman-Kac formula under the G-framework (see Theorem 2.3), which built the relationship between $G$-FBSDEs and fully nonlinear partial differential equations (PDEs). In addition, Cheridito et al. [6] and Soner et al. [31] developed a new type of fully nonlinear FBSDEs, called the 2FBSDEs, which is also associated with a class of fully nonlinear PDEs. Tremendous efforts have been made on the numerical computation of FBSDEs or 2FBSDEs (see, e.g., [2, 3, 4, 5, 7, 12, 13, 14, 15, 16, 21, 24, 25, 30, 32, 34, 35, 36, 37] and the references therein), but little seems to be known about the numerical results for $G$-FBSDEs. There are some obstacles in developing the numerical scheme for $G$-FBSDEs. On the one hand, there is no density representation for the $G$-normal distribution, and the classical numerical integration methods are no longer applicable. On the other hand, owing to the sublinear nature of $G$-expectation, many conclusions under the linear expectation can not be extended to the $G$-expectation, which increases the difficulty of theoretical analysis.

For the first obstacle, inspired by the $G$-expectation representation (see Theorem 2.2), we give an approximate conditional $G$-expectation, which leads to the feasible methods to calculate the distribution of $G$-Brownian motion, including the trinomial tree rule and the 
some efficient numerical schemes for solving $G$-FBSDEs are proposed. For the second obstacle, using the property of $G$-expectation, we rigorously analyze the errors of the proposed schemes and prove the convergence. Some examples are given to numerically demonstrate the accuracy of the proposed schemes. To the best of our knowledge, this is the first attempt to design the numerical scheme for $G$-FBSDEs.

The paper is organized as follow. In Section 2, we recall some preliminaries used in the $G$-framework. We propose the numerical schemes for $G$-FBSDEs in Section 3. The convergence result of the proposed schemes is rigorously proved in Section 4. In Section 5, we extend our result to the case of multi-dimensional Brownian motion. Finally, various numerical examples illustrate the performance of our schemes in Section 6.

\section{Preliminaries}

For any fixed $T>0$, let $\Omega_{T}=C_{0}\left([0, T] ; \mathbb{R}^{d}\right)$ be the space of $\mathbb{R}^{d}$-valued continuous paths on $[0, T]$ with $\omega_{0}=0$, endowed with the supremum norm, and $B_{t}(\omega)=\omega_{t}$ be the canonical process. Set

$$
\operatorname{Lip}\left(\Omega_{T}\right)=\left\{\varphi\left(B_{t_{1}}, \ldots, B_{t_{n}}\right): n \geq 1, t_{1}, \ldots, t_{n} \in[0, T], \varphi \in C_{b, L i p}\left(\mathbb{R}^{d \times n}\right)\right\}
$$

where $C_{b, L i p}\left(\mathbb{R}^{d \times n}\right)$ denotes the set of bounded Lipschitz functions on $\mathbb{R}^{d \times n}$.

Peng [27] constructed a consistent sublinear expectation space $\left(\Omega_{T}, \operatorname{Lip}\left(\Omega_{T}\right), \hat{\mathbb{E}}_{,}\left(\hat{\mathbb{E}}_{t}\right)_{t \geq 0}\right)$, called a $G$-expectation space, and the canonical process $\left(B_{t}\right)_{t \geq 0}$ is called a $G$-Brownian motion. The monotonic and sublinear function $G: \mathbb{S}(d) \rightarrow \mathbb{R}$ is defined by

$$
G(A):=\frac{1}{2} \hat{\mathbb{E}}\left[\left\langle A B_{1}, B_{1}\right\rangle\right], \quad A \in \mathbb{S}(d),
$$

where $\mathbb{S}(d)$ denotes the collection of $d \times d$ symmetric matrices. Note that there exists a bounded and closed subset $\Sigma \subset \mathbb{S}^{+}(d)$ such that

$$
G(A)=\frac{1}{2} \sup _{Q \in \Sigma} \operatorname{tr}[Q A], \quad A \in \mathbb{S}(d),
$$

where $\mathbb{S}^{+}(d)$ denotes the collection of nonnegative definite elements in $\mathbb{S}(d)$. In this paper, we assume that $G$ is non-degenerate, i.e., there exist some constants $0<\underline{\sigma}^{2} \leq \bar{\sigma}^{2}<\infty$ such that $\frac{1}{2} \underline{\sigma}^{2} \operatorname{tr}[A-B] \leq G(A)-G(B) \leq \frac{1}{2} \bar{\sigma}^{2} \operatorname{tr}[A-B]$ for $A \geq B$.

For each given $p \geq 1$, define $\|X\|_{L_{G}^{p}}=\left(\hat{\mathbb{E}}\left[|X|^{p}\right]\right)^{1 / p}$ for $X \in \operatorname{Lip}\left(\Omega_{T}\right)$, and denote by $L_{G}^{p}\left(\Omega_{T}\right)$ the completion of $\operatorname{Lip}\left(\Omega_{T}\right)$ under the norm $\|\cdot\|_{L_{G}^{p}}$. Then for $t \in[0, T]$, $\hat{\mathbb{E}}_{t}[\cdot]$ can be extended continuously to $L_{G}^{1}\left(\Omega_{T}\right)$. 
Definition 2.1. Let $M_{G}^{0}(0, T)$ be the collection of processes in the following form: for a given partition $\left\{t_{0}, t_{1}, \ldots, t_{N}\right\}$ of $[0, T]$,

$$
\eta_{t}(\omega)=\sum_{n=0}^{N-1} \xi_{n}(\omega) I_{\left[t_{n}, t_{n+1}\right)}(t),
$$

where $\xi_{n} \in \operatorname{Lip}\left(\Omega_{t_{n}}\right), n=0,1, \ldots, N-1$.

Theorem $2.2([\mathbf{1 0}, \mathbf{1 7}])$. There exists a weakly compact family $\mathcal{P}$ of probability measures on $\left(\Omega_{T}, \mathcal{B}\left(\Omega_{T}\right)\right)$ such that

$$
\hat{\mathbb{E}}[X]=\sup _{P \in \mathcal{P}} \mathbb{E}_{P}[X], \text { for all } X \in L_{G}^{1}\left(\Omega_{T}\right) .
$$

$\mathcal{P}$ is called a set that represents $\hat{\mathbb{E}}$.

Let $\left(X_{s}^{t, x}, Y_{s}^{t, x}, Z_{s}^{t, x}, K_{s}^{t, x}\right)$, for $s \in[t, T]$, be the solution of (1.1) starting from $t$ with $X_{t}=x$, that is,

$$
\begin{aligned}
X_{s}^{t, x}= & x+\int_{t}^{s} b\left(r, X_{r}^{t, x}\right) d r+\int_{t}^{s} h_{i j}\left(r, X_{r}^{t, x}\right) d\left\langle B^{i}, B^{j}\right\rangle_{r}+\int_{t}^{s} \sigma\left(r, X_{r}^{t, x}\right) d B_{r}, \\
Y_{s}^{t, x}= & \phi\left(X_{T}^{t, x}\right)+\int_{s}^{T} f\left(r, X_{r}^{t, x}, Y_{r}^{t, x}, Z_{r}^{t, x}\right) d r+\int_{s}^{T} g_{i j}\left(r, X_{r}^{t, x}, Y_{r}^{t, x}, Z_{r}^{t, x}\right) d\left\langle B^{i}, B^{j}\right\rangle_{r} \\
& -\int_{s}^{T} Z_{r}^{t, x} d B_{r}-\left(K_{T}^{t, x}-K_{s}^{t, x}\right) .
\end{aligned}
$$

Next, we shall present the nonlinear Feynman-Kac formula under the $G$-framework.

Theorem 2.3 ([20]). Assume that the functions $b, h_{i j}, \sigma, f, g_{i j}$, and $\phi$ are uniformly Lipschitz continuous with respect to $(x, y, z)$ and continuous with respect to $t$, and $h_{i j}=h_{j i}$, $g_{i j}=g_{j i}$ for $1 \leq i, j \leq d$. Let $u(t, x):=Y_{t}^{t, x}$ for $(t, x) \in[0, T] \times \mathbb{R}^{m}$. Then $u(t, x)$ is the unique solution of the following PDE:

$$
\left\{\begin{array}{l}
\partial_{t} u+F\left(D_{x}^{2} u, D_{x} u, u, x, t\right)=0 \\
u(T, x)=\phi(x)
\end{array}\right.
$$


where

$$
\begin{aligned}
F\left(D_{x}^{2} u, D_{x} u, u, x, t\right)= & G\left(H\left(D_{x}^{2} u, D_{x} u, u, x, t\right)\right)+\left\langle b(t, x), D_{x} u\right\rangle \\
+ & f\left(t, x, u,\left\langle\sigma_{1}(t, x), D_{x} u\right\rangle, \ldots,\left\langle\sigma_{d}(t, x), D_{x} u\right\rangle\right), \\
H_{i j}\left(D_{x}^{2} u, D_{x} u, u, x, t\right)= & \left\langle D_{x}^{2} u \sigma_{i}(t, x), \sigma_{j}(t, x)\right\rangle+2\left\langle D_{x} u, h_{i j}(t, x)\right\rangle \\
& +2 g_{i j}\left(t, x, u,\left\langle\sigma_{1}(t, x), D_{x} u\right\rangle, \ldots,\left\langle\sigma_{d}(t, x), D_{x} u\right\rangle\right) .
\end{aligned}
$$

Remark 2.4. By Theorem 2.3, the solution of the G-FBSDE (1.1) can be represented as

$$
\begin{aligned}
Y_{t}= & u\left(t, X_{t}\right), \quad Z_{t}=D_{x} u\left(t, X_{t}\right)^{\top} \sigma\left(t, X_{t}\right), \\
K_{t}= & \frac{1}{2} \int_{0}^{t} H_{i j}\left(D_{x}^{2} u\left(s, X_{s}\right), D_{x} u\left(s, X_{s}\right), u\left(s, X_{s}\right), X_{s}, s\right) d\left\langle B^{i}, B^{j}\right\rangle_{s} \\
& -\int_{0}^{t} G\left(H\left(D_{x}^{2} u\left(s, X_{s}\right), D_{x} u\left(s, X_{s}\right), u\left(s, X_{s}\right), X_{s}, s\right)\right) d s .
\end{aligned}
$$

For readers' convenience, we list the main notations of this paper as follows.

- $\Delta B_{n+1}:=B_{t_{n+1}}-B_{t_{n}}, \quad \Delta\langle B\rangle_{n+1}:=\langle B\rangle_{t_{n+1}}-\langle B\rangle_{t_{n}}$;

- $\hat{\mathbb{E}}_{t}^{x}[\eta]:=\hat{\mathbb{E}}\left[\eta \mid X_{t}=x\right]$, the conditional $G$-expectation of the random variable $\eta$;

- $\mathbb{E}_{t}^{\sigma, x}[\eta]:=\mathbb{E}_{P^{\sigma}}\left[\eta \mid X_{t}=x\right]$, the conditional expectation of the random variable $\eta$ under the probability measure $P^{\sigma} \in \mathcal{P}$;

- $\tilde{\mathbb{E}}_{t}^{x}[\eta]:=\sup _{\sigma \in\{\underline{\sigma}, \bar{\sigma}\}} \mathbb{E}_{t}^{\sigma, x}[\eta]$, the approximate conditional $G$-expectation of the random variable $\eta$;

- $\tilde{\mathbb{E}}^{T R}\left[\varphi\left(B_{t},\langle B\rangle_{t}\right)\right]:=\sup _{\sigma \in\{\underline{\sigma}, \bar{\sigma}\}} \sum_{i=1}^{3} \omega_{i}^{\sigma} \varphi\left(\sqrt{t} q_{i}, t q_{i}^{2}\right)$, the trinomial tree rule approximation for the distribution of $\varphi\left(B_{t},\langle B\rangle_{t}\right)$ with the weights $\left\{\omega_{i}^{\sigma}\right\}_{i=1}^{3}$ and the nodes $\left\{q_{i}\right\}_{i=1}^{3}$.

\section{Numerical schemes for $G$-FBSDEs}

We first consider the one-dimensional $G$-Brownian motion case. The results for the multidimensional $G$-Brownian motion case will be given in Section 5. For the time interval $[0, T]$, we introduce a uniform time partition $0=t_{0}<t_{1}<\cdots<t_{N}=T$ with $\Delta t=t_{n+1}-t_{n}=$ $T / N$. We use the following Euler scheme to approximate the $G$-SDE (2.1):

$$
X^{n+1}=X^{n}+b\left(t_{n}, X^{n}\right) \Delta t+h\left(t_{n}, X^{n}\right) \Delta\langle B\rangle_{n+1}+\sigma\left(t_{n}, X^{n}\right) \Delta B_{n+1},
$$

and $X^{0}=x_{0}$, for $n=0, \ldots, N-1$, where $\Delta B_{n+1} \sim N(0, \Delta t \Sigma)$ and $\Delta\langle B\rangle_{n+1} \sim N(\Delta t \Sigma, 0)$ with $\Sigma=\left[\underline{\sigma}^{2}, \bar{\sigma}^{2}\right]$. 
Let $\left(X_{t}^{t_{n}, X^{n}}, Y_{t}^{t_{n}, X^{n}}, Z_{t}^{t_{n}, X^{n}}, K_{t}^{t_{n}, X^{n}}\right)$, for $t \in\left[t_{n}, T\right]$, be the solution of (2.1)-(2.2) with $(t, x)=\left(t_{n}, X^{n}\right)$, and denote $f_{t}^{t_{n}, X^{n}}=f\left(t, X_{t}^{t_{n}, X^{n}}, Y_{t}^{t_{n}, X^{n}}, Z_{t}^{t_{n}, X^{n}}\right)$ and $g_{t}^{t_{n}, X^{n}}=g\left(t, X_{t}^{t_{n}, X^{n}}\right.$, $\left.Y_{t}^{t_{n}, X^{n}}, Z_{t}^{t_{n}, X^{n}}\right)$. Then, for $n=0,1, \ldots, N-1$,

$$
\begin{aligned}
& X_{t_{n+1}}^{t_{n}, X^{n}}=X^{n}+\int_{t_{n}}^{t_{n+1}} b\left(t, X_{t}^{t_{n}, X^{n}}\right) d t+\int_{t_{n}}^{t_{n+1}} h\left(t, X_{t}^{t_{n}, X^{n}}\right) d\langle B\rangle_{t}+\int_{t_{n}}^{t_{n+1}} \sigma\left(t, X_{t}^{t_{n}, X^{n}}\right) d B_{t}, \\
& Y_{t_{n}}^{t_{n}, X^{n}}=Y_{t_{n+1}}^{t_{n}, X^{n}}+\int_{t_{n}}^{t_{n+1}} f_{t}^{t_{n}, X^{n}} d t+\int_{t_{n}}^{t_{n+1}} g_{t}^{t_{n}, X^{n}} d\langle B\rangle_{t}-\int_{t_{n}}^{t_{n+1}} Z_{t}^{t_{n}, X^{n}} d B_{t}-\left(K_{t_{n+1}}^{t_{n}, X^{n}}-K_{t_{n}}^{t_{n}, X^{n}}\right) .
\end{aligned}
$$

\subsection{Conditional $G$-expectation approximation}

Taking the conditional $G$-expectation $\hat{\mathbb{E}}_{t_{n}}^{X^{n}}[\cdot]:=\hat{\mathbb{E}}\left[\cdot \mid X_{t_{n}}=X^{n}\right]$ on (3.2) and noting that $K_{t}$ is a $G$-martingale, we have

$$
Y_{t_{n}}^{t_{n}, X^{n}}=\hat{\mathbb{E}}_{t_{n}}^{X^{n}}\left[Y_{t_{n+1}}^{t_{n}, X^{n}}+\int_{t_{n}}^{t_{n+1}} f_{t}^{t_{n}, X^{n}} d t+\int_{t_{n}}^{t_{n+1}} g_{t}^{t_{n}, X^{n}} d\langle B\rangle_{t}\right] .
$$

Using the right rectangle formula in (3.3) and approximating the forward process, we obtain

$$
\begin{aligned}
Y_{t_{n}}^{t_{n}, X^{n}} & =\hat{\mathbb{E}}_{t_{n}}^{X^{n}}\left[Y_{t_{n+1}}^{t_{n}, X^{n}}+f_{t_{n+1}}^{t_{n}, X^{n}} \Delta t+g_{t_{n+1}}^{t_{n}, X^{n}} \Delta\langle B\rangle_{n+1}\right]+\hat{R}_{y_{1}}^{n} \\
& =\hat{\mathbb{E}}_{t_{n}}^{X^{n}}\left[Y_{t_{n+1}}^{t_{n+1}, X^{n+1}}+f_{t_{n+1}}^{t_{n+1}, X^{n+1}} \Delta t+g_{t_{n+1}}^{t_{n+1}, X^{n+1}} \Delta\langle B\rangle_{n+1}\right]+\hat{R}_{y_{1}}^{n}+\hat{R}_{y_{2}}^{n}
\end{aligned}
$$

where

$$
\begin{aligned}
\hat{R}_{y_{1}}^{n}= & \hat{\mathbb{E}}_{t_{n}}^{X^{n}}\left[Y_{t_{n+1}}^{t_{n}, X^{n}}+\int_{t_{n}}^{t_{n+1}} f_{t}^{t_{n}, X^{n}} d t+\int_{t_{n}}^{t_{n+1}} g_{t}^{t_{n}, X^{n}} d\langle B\rangle_{t}\right] \\
& -\hat{\mathbb{E}}_{t_{n}}^{X^{n}}\left[Y_{t_{n+1}}^{t_{n}, X^{n}}+f_{t_{n+1}}^{t_{n}, X^{n}} \Delta t+g_{t_{n+1}}^{t_{n}, X^{n}} \Delta\langle B\rangle_{n+1}\right],
\end{aligned}
$$

and

$$
\begin{aligned}
\hat{R}_{y_{2}}^{n}= & \hat{\mathbb{E}}_{t_{n} X^{n}}^{n}\left[Y_{t_{n+1}}^{t_{n}, X^{n}}+f_{t_{n+1}}^{t_{n}, X^{n}} \Delta t+g_{t_{n+1}}^{t_{n}, X^{n}} \Delta\langle B\rangle_{n+1}\right] \\
& -\hat{\mathbb{E}}_{t_{n}}^{X^{n}}\left[Y_{t_{n+1}}^{t_{n+1}, X^{n+1}}+f_{t_{n+1}}^{t_{n+1}, X^{n+1}} \Delta t+g_{t_{n+1}}^{t_{n+1}, X^{n+1}} \Delta\langle B\rangle_{n+1}\right] .
\end{aligned}
$$

For any $\eta \in L_{G}^{1}\left(\Omega_{T}\right)$, define the approximate conditional $G$-expectation $\tilde{\mathbb{E}}_{t_{n}}^{X^{n}}[\eta]$ by

$$
\tilde{\mathbb{E}}_{t_{n}}^{X^{n}}[\eta]:=\sup _{\sigma \in\{\underline{\sigma}, \bar{\sigma}\}} \mathbb{E}_{t_{n}}^{\sigma, X^{n}}[\eta]:=\sup _{\sigma \in\{\underline{\sigma}, \bar{\sigma}\}} \mathbb{E}_{P^{\sigma}}\left[\eta \mid X_{t_{n}}=X^{n}\right]
$$

where $P^{\sigma} \in \mathcal{P}$ is a probability measure, under which $\left(B_{t}\right)_{t \geq 0}$ is the classical Brownian motion with $\mathbb{E}_{P^{\sigma}}\left[B_{t}\right]=0$ and $\mathbb{E}_{P^{\sigma}}\left[B_{t}^{2}\right]=\sigma^{2} t$. Then we have

$$
Y_{t_{n}}^{t_{n}, X^{n}}=\tilde{\mathbb{E}}_{t_{n}}^{X^{n}}\left[Y_{t_{n+1}}^{t_{n+1}, X^{n+1}}+f_{t_{n+1}}^{t_{n+1}, X^{n+1}} \Delta t+g_{t_{n+1}}^{t_{n+1}, X^{n+1}} \Delta\langle B\rangle_{n+1}\right]+\tilde{R}_{y}^{n}
$$


where $\tilde{R}_{y}^{n}=\hat{R}_{y_{1}}^{n}+\hat{R}_{y_{2}}^{n}+\tilde{R}_{y_{3}}^{n}$, with

$$
\begin{aligned}
\tilde{R}_{y_{3}}^{n}= & \hat{\mathbb{E}}_{t_{n}}^{X^{n}}\left[Y_{t_{n+1}}^{t_{n+1}, X^{n+1}}+f_{t_{n+1}}^{t_{n+1}, X^{n+1}} \Delta t+g_{t_{n+1}}^{t_{n+1}, X^{n+1}} \Delta\langle B\rangle_{n+1}\right] \\
& -\tilde{\mathbb{E}}_{t_{n}}^{X^{n}}\left[Y_{t_{n+1}}^{t_{n+1}, X^{n+1}}+f_{t_{n+1}}^{t_{n+1}, X^{n+1}} \Delta t+g_{t_{n+1}}^{t_{n+1}, X^{n+1}} \Delta\langle B\rangle_{n+1}\right] .
\end{aligned}
$$

Now let us multiply both sides of (3.2) by $\Delta B_{n+1}$ and take the conditional expectation $\mathbb{E}_{t_{n}}^{\sigma, X^{n}}[\cdot]$, for any $\sigma \in\{\underline{\sigma}, \bar{\sigma}\}$, then we obtain

$$
\mathbb{E}_{t_{n}}^{\sigma, X^{n}}\left[\int_{t_{n}}^{t_{n+1}} Z_{t}^{t_{n}, X^{n}} d B_{t} \Delta B_{n+1}\right]=\mathbb{E}_{t_{n}}^{\sigma, X^{n}}\left[Y_{t_{n+1}}^{t_{n+1}, X^{n+1}} \Delta B_{n+1}\right]+R_{z_{1}}^{n, \sigma}+R_{z_{2}}^{n, \sigma}
$$

where

$$
\begin{aligned}
R_{z_{1}}^{n, \sigma}= & \mathbb{E}_{t_{n}}^{\sigma, X^{n}}\left[\int_{t_{n}}^{t_{n+1}} f_{t}^{t_{n}, X^{n}} d t \Delta B_{n+1}+\int_{t_{n}}^{t_{n+1}} g_{t}^{t_{n}, X^{n}} d\langle B\rangle_{t} \Delta B_{n+1}\right] \\
& -\mathbb{E}_{t_{n}}^{\sigma, X^{n}}\left[\left(K_{t_{n+1}}^{t_{n}, X^{n}}-K_{t_{n}}^{t_{n}, X^{n}}\right) \Delta B_{n+1}\right], \\
R_{z_{2}}^{n, \sigma}= & \mathbb{E}_{t_{n}}^{\sigma, X^{n}}\left[Y_{t_{n+1}}^{t_{n}, X^{n}} \Delta B_{n+1}\right]-\mathbb{E}_{t_{n}}^{\sigma, X^{n}}\left[Y_{t_{n+1}}^{t_{n+1}, X^{n+1}} \Delta B_{n+1}\right] .
\end{aligned}
$$

Noting that $B_{t} \sim N\left(0, \sigma^{2} t\right)$ under $\mathbb{E}^{\sigma}$, by the Itô isometry formula, we have

$$
\mathbb{E}_{t_{n}}^{\sigma, X^{n}}\left[\int_{t_{n}}^{t_{n+1}} Z_{t}^{t_{n}, X^{n}} d B_{t} \Delta B_{n+1}\right]=\sigma^{2} \mathbb{E}_{t_{n}}^{\sigma, X^{n}}\left[\int_{t_{n}}^{t_{n+1}} Z_{t}^{t_{n}, X^{n}} d t\right] .
$$

Combining (3.10) and (3.13), for $\sigma \in\{\underline{\sigma}, \bar{\sigma}\}$, we have

$$
\sigma^{2} \Delta t Z_{t_{n}}^{t_{n}, X^{n}}=\mathbb{E}_{t_{n}}^{\sigma, X^{n}}\left[Y_{t_{n+1}}^{t_{n+1}, X^{n+1}} \Delta B_{n+1}\right]+\tilde{R}_{z}^{n, \sigma}
$$

where $\tilde{R}_{z}^{n, \sigma}=R_{z_{1}}^{n, \sigma}+R_{z_{2}}^{n, \sigma}-R_{z_{3}}^{n, \sigma}$ and

$$
R_{z_{3}}^{n, \sigma}=\sigma^{2} \mathbb{E}_{t_{n}}^{\sigma, X^{n}}\left[\int_{t_{n}}^{t_{n+1}} Z_{t}^{t_{n}, X^{n}} d t\right]-\sigma^{2} \Delta t Z_{t_{n}}^{t_{n}, X^{n}}
$$

Thus we obtain

$$
\begin{aligned}
& Y_{t_{n}}^{t_{n}, X^{n}}=\tilde{\mathbb{E}}_{t_{n}}^{X^{n}}\left[Y_{t_{n+1}}^{t_{n+1}, X^{n+1}}+f_{t_{n+1}}^{t_{n+1}, X^{n+1}} \Delta t+g_{t_{n+1}}^{t_{n+1}, X^{n+1}} \Delta\langle B\rangle_{n+1}\right]+\tilde{R}_{y}^{n}, \\
& \sigma^{2} \Delta t Z_{t_{n}}^{t_{n}, X^{n}}=\mathbb{E}_{t_{n}}^{\sigma, X^{n}}\left[Y_{t_{n+1}}^{t_{n+1}, X^{n+1}} \Delta B_{n+1}\right]+\tilde{R}_{z}^{n, \sigma},
\end{aligned}
$$

for any $\sigma \in\{\underline{\sigma}, \bar{\sigma}\}$. 


\subsection{Trinomial tree rule}

Notice that $Y_{t_{n+1}}^{t_{n+1}, X^{n+1}}, f_{t_{n+1}}^{t_{n+1}, X^{n+1}}, g_{t_{n+1}}^{t_{n+1}, X^{n+1}} \Delta\langle B\rangle_{n+1}$, and $Y_{t_{n+1}}^{t_{n+1}, X^{n+1}} \Delta B_{n+1}$ in (3.16)(3.17) are the functions of $X^{n+1}, \Delta B_{n+1}$, and $\Delta\langle B\rangle_{n+1}$. For a function $\varphi: \mathbb{R}^{m} \times \mathbb{R} \times \mathbb{R} \rightarrow \mathbb{R}$, denote $\varphi_{t_{n+1}}=\varphi\left(X^{n+1}, \Delta B_{n+1}, \Delta\langle B\rangle_{n+1}\right)$, we define the following trinomial tree rule to approximate $\tilde{\mathbb{E}}_{t_{n}}^{X^{n}}\left[\varphi_{t_{n+1}}\right]$ :

$$
\tilde{\mathbb{E}}_{t_{n}}^{T R, X^{n}}\left[\varphi_{t_{n+1}}\right]:=\sup _{\sigma \in\{\underline{\sigma}, \bar{\sigma}\}} \mathbb{E}_{t_{n}}^{T R, \sigma, X^{n}}\left[\varphi_{t_{n+1}}\right]:=\sup _{\sigma \in\{\sigma, \bar{\sigma}\}} \sum_{i=1}^{3} \omega_{i}^{\sigma} \tilde{\varphi}\left(X^{n}, \sqrt{\Delta t} q_{i}, \Delta t q_{i}^{2}\right),
$$

where $\tilde{\varphi}(x, y, z)=\varphi\left(x+b\left(t_{n}, x\right) \Delta t+\sigma\left(t_{n}, x\right) y+h\left(t_{n}, x\right) z, y, z\right)$ and

$$
\begin{cases}q_{1}=-1, & \omega_{1}^{\sigma}=\sigma^{2} / 2 ; \\ q_{2}=0, & \omega_{2}^{\sigma}=1-\sigma^{2} ; \\ q_{3}=1, & \omega_{3}^{\sigma}=\sigma^{2} / 2 .\end{cases}
$$

More precisely, we define the associated discrete sublinear expectation

$$
\tilde{\mathbb{E}}^{T R}\left[\varphi_{t_{n+1}}\right]=\tilde{\mathbb{E}}_{t_{0}}^{T R, x_{0}}\left[\tilde{\mathbb{E}}_{t_{1}}^{T R, X^{1}}\left[\cdots \tilde{\mathbb{E}}_{t_{n}}^{T R, X^{n}}\left[\varphi_{t_{n+1}}\right]\right] .\right.
$$

Remark 3.1. From the definition of $\left\{\omega_{i}^{\sigma}\right\}_{i=1}^{3}$ and $\left\{q_{i}\right\}_{i=1}^{3}$, one can check that

$$
\sum_{i=1}^{3} \omega_{i}^{\sigma}=1, \quad \sum_{i=1}^{3} \omega_{i}^{\sigma} q_{i}=0, \quad \sum_{i=1}^{3} \omega_{i}^{\sigma} q_{i}^{2}=\sigma^{2} .
$$

It is easy to verify the following properties.

Proposition 3.2. Assume that $\varphi_{t_{n+1}}=\varphi\left(X^{n+1}, \Delta B_{n+1}, \Delta\langle B\rangle_{n+1}\right)$. Then

(a) $\tilde{\mathbb{E}}^{T R}\left[\tilde{\mathbb{E}}_{t_{n}}^{T R, X^{n}}\left[\varphi_{t_{n+1}}\right]\right]=\tilde{\mathbb{E}}^{T R}\left[\varphi_{t_{n+1}}\right]$;

(b) $\left|\mathbb{E}_{t_{n}}^{T R, \sigma, X^{n}}\left[\varphi_{t_{n+1}}\right]\right|^{2} \leq \mathbb{E}_{t_{n}}^{T R, \sigma, X^{n}}\left[\left|\varphi_{t_{n+1}}\right|^{2}\right]$;

(c) $\left|\mathbb{E}_{t_{n}}^{T R, \sigma, X^{n}}\left[\varphi_{t_{n+1}} \Delta B_{n+1}\right]\right|^{2} \leq\left(\mathbb{E}_{t_{n}}^{T R, \sigma, X^{n}}\left[\left|\varphi_{t_{n+1}}\right|^{2}\right]-\left|\mathbb{E}_{t_{n}}^{T R, \sigma, X^{n}}\left[\varphi_{t_{n+1}}\right]\right|^{2}\right) \sigma^{2} \Delta t$.

From the definitions of $\tilde{\mathbb{E}}_{t_{n}}^{T R, X^{n}}[\cdot]$ and $\mathbb{E}_{t_{n}}^{T R, \sigma, X^{n}}[\cdot]$, we have the following approximations:

$$
\begin{aligned}
& \tilde{\mathbb{E}}_{t_{n}}^{X^{n}}\left[Y_{t_{n+1}}^{t_{n+1}, X^{n+1}}+f_{t_{n+1}}^{t_{n+1}, X^{n+1}} \Delta t+g_{t_{n+1}}^{t_{n+1}, X^{n+1}} \Delta\langle B\rangle_{n+1}\right] \\
& =\tilde{\mathbb{E}}_{t_{n}}^{T R, X^{n}}\left[Y_{t_{n+1}}^{t_{n+1}, X^{n+1}}+f_{t_{n+1}}^{t_{n+1}, X^{n+1}} \Delta t+g_{t_{n+1}}^{t_{n+1}, X^{n+1}} \Delta\langle B\rangle_{n+1}\right]+R_{y}^{T, n},
\end{aligned}
$$

and

$$
\mathbb{E}_{t_{n}}^{\sigma, X^{n}}\left[Y_{t_{n+1}}^{t_{n+1}, X^{n+1}} \Delta B_{n+1}\right]=\mathbb{E}_{t_{n}}^{T R, \sigma, X^{n}}\left[Y_{t_{n+1}}^{t_{n+1}, X^{n+1}} \Delta B_{n+1}\right]+R_{z}^{T, n, \sigma},
$$


where the errors

$$
\begin{aligned}
R_{y}^{T, n}= & \tilde{\mathbb{E}}_{t_{n}}^{X^{n}}\left[Y_{t_{n+1}}^{t_{n+1}, X^{n+1}}+f_{t_{n+1}}^{t_{n+1}, X^{n+1}} \Delta t+g_{t_{n+1}}^{t_{n+1}, X^{n+1}} \Delta\langle B\rangle_{n+1}\right] \\
& -\tilde{\mathbb{E}}_{t_{n}}^{T R, X^{n}}\left[Y_{t_{n+1}}^{t_{n+1}, X^{n+1}}+f_{t_{n+1}}^{t_{n+1}, X^{n+1}} \Delta t+g_{t_{n+1}}^{t_{n+1}, X^{n+1}} \Delta\langle B\rangle_{n+1}\right], \\
R_{z}^{T, n, \sigma}= & \mathbb{E}_{t_{n}}^{\sigma, X^{n}}\left[Y_{t_{n+1}}^{t_{n+1}, X^{n+1}} \Delta B_{n+1}\right]-\mathbb{E}_{t_{n}}^{T R, \sigma, X^{n}}\left[Y_{t_{n+1}}^{t_{n+1}, X^{n+1}} \Delta B_{n+1}\right] .
\end{aligned}
$$

Based on (3.16), (3.17), (3.20), and (3.21), for $\sigma \in\{\underline{\sigma}, \bar{\sigma}\}$, we obtain the following reference equations

$$
\begin{aligned}
& Y_{t_{n}, X^{n}}^{t_{n}}=\tilde{\mathbb{E}}_{t_{n}}^{T R, X^{n}}\left[Y_{t_{n+1}}^{t_{n+1}, X^{n+1}}+f_{t_{n+1}}^{t_{n+1}, X^{n+1}} \Delta t+g_{t_{n+1}}^{t_{n+1}, X^{n+1}} \Delta\langle B\rangle_{n+1}\right]+\tilde{R}_{y}^{n}+R_{y}^{T, n}, \\
& \sigma^{2} \Delta t Z_{t_{n}}^{t_{n}, X^{n}}=\mathbb{E}_{t_{n}}^{T R, \sigma, X^{n}}\left[Y_{t_{n+1}}^{t_{n+1}, X^{n+1}} \Delta B_{n+1}\right]+\tilde{R}_{z}^{n, \sigma}+R_{z}^{T, n, \sigma} .
\end{aligned}
$$

\subsection{The discrete scheme}

Let $Y^{n}$ and $Z^{n}$ be the numerical approximations for the solutions $Y_{t}$ and $Z_{t}$ of the $G$ FBSDE (1.1) at time $t_{n}$, respectively, and denote $f^{n+1}=f\left(t_{n+1}, X^{n+1}, Y^{n+1}, Z^{n+1}\right), g^{n+1}=$ $g\left(t_{n+1}, X^{n+1}, Y^{n+1}, Z^{n+1}\right)$. Based on the reference equations (3.24)-(3.25), we propose the following numerical scheme for solving the $G$-FBSDE (1.1):

Scheme 1. Given random variables $Y^{N}$ and $Z^{N}$, for $n=N-1, \ldots, 0$ and $\sigma \in\{\underline{\sigma}, \bar{\sigma}\}$, solve random variables $Y^{n}=Y^{n}\left(X^{n}\right)$ and $Z^{n}=Z^{n}\left(X^{n}\right)$ from

$$
\begin{aligned}
Y^{n} & =\tilde{\mathbb{E}}_{t_{n}}^{T R, X^{n}}\left[Y^{n+1}+f^{n+1} \Delta t+g^{n+1} \Delta\langle B\rangle_{n+1}\right], \\
Z^{n} & =\mathbb{E}_{t_{n}}^{T R, \sigma, X^{n}}\left[Y^{n+1} \Delta B_{n+1}\right] / \sigma^{2} \Delta t,
\end{aligned}
$$

with

$$
X^{n+1}=X^{n}+b\left(t_{n}, X^{n}\right) \Delta t+h\left(t_{n}, X^{n}\right) \Delta\langle B\rangle_{n+1}+\sigma\left(t_{n}, X^{n}\right) \Delta B_{n+1} .
$$

Noticed that when solving for $Z^{n}, \mathbb{E}_{t_{n}}^{T R, \sigma, X^{n}}[\cdot]$ in (3.27) can be selected arbitrarily among $\sigma \in\{\underline{\sigma}, \bar{\sigma}\}$. Besides, the parameter $\sigma$ that $\tilde{\mathbb{E}}_{t_{n}}^{T R, X^{n}}[\cdot]$ in (3.26) reaches its maximum can be obtained in the numerical test, denoted as $\sigma^{n}$. So one natural choice for solving $Z^{n}$ is to let $\sigma=\sigma^{n}$. In this case, Scheme 1 becomes

Scheme 2. Given random variables $Y^{N}$ and $Z^{N}$, for $n=N-1, \ldots, 0$, solve random variables $Y^{n}=Y^{n}\left(X^{n}\right)$ and $Z^{n}=Z^{n}\left(X^{n}\right)$ from

$$
\begin{aligned}
Y^{n} & =\mathbb{E}_{t_{n}}^{T R, \sigma^{n}, X^{n}}\left[Y^{n+1}+f^{n+1} \Delta t+g^{n+1} \Delta\langle B\rangle_{n+1}\right], \\
Z^{n} & =\mathbb{E}_{t_{n}}^{T R, \sigma^{n}, X^{n}}\left[Y^{n+1} \Delta B_{n+1}\right] /\left(\sigma^{n}\right)^{2} \Delta t,
\end{aligned}
$$

with

$$
X^{n+1}=X^{n}+b\left(t_{n}, X^{n}\right) \Delta t+h\left(t_{n}, X^{n}\right) \Delta\langle B\rangle_{n+1}+\sigma\left(t_{n}, X^{n}\right) \Delta B_{n+1} .
$$


In fact, Scheme 1 is independent of the parameter $\sigma$ when solving $Z^{n}$ (see Lemma 4.4). Therefore, Scheme 1 and Scheme 2 are essentially the same. In order to simplify the presentation, in the following, we will only perform the convergence analysis for Scheme 2 .

\section{Convergence analysis}

In this section, we focus on the convergence analysis of our discrete schemes. In the sequel, $C$ represents a generic constant which does not depend on the time partition and may be different from line to line.

For $n=0,1, \ldots, N-1$, assume that $\tilde{\mathbb{E}}_{t_{n}}^{T R, X^{n}}$ in $(3.24)$ reaches its maximum at $\mathbb{E}_{t_{n}}^{T R, \tilde{\sigma}^{n}, X^{n}}$ with the parameter $\tilde{\sigma}^{n}$ and let $\sigma=\tilde{\sigma}^{n}$ in (3.25), that is,

$$
\begin{aligned}
& Y_{t_{n}}^{t_{n}, X^{n}}=\mathbb{E}_{t_{n}}^{T R, \tilde{\sigma}^{n}, X^{n}}\left[Y_{t_{n+1}}^{t_{n+1}, X^{n+1}}+f_{t_{n+1}}^{t_{n+1}, X^{n+1}} \Delta t+g_{t_{n+1}}^{t_{n+1}, X^{n+1}} \Delta\langle B\rangle_{n+1}\right]+\tilde{R}_{y}^{n}+R_{y}^{G, n}, \\
& \left(\tilde{\sigma}^{n}\right)^{2} \Delta t Z_{t_{n}}^{t_{n}, X^{n}}=\mathbb{E}_{t_{n}}^{T R, \tilde{\sigma}^{n}, X^{n}}\left[Y_{t_{n+1}}^{t_{n+1}, X^{n+1}} \Delta B_{n+1}\right]+\tilde{R}_{z}^{n, \tilde{\sigma}^{n}}+R_{z}^{G, n, \tilde{\sigma}^{n}} .
\end{aligned}
$$

Define the related process $\tilde{Z}^{n}$ as follows

$$
\tilde{Z}^{n}=\mathbb{E}_{t_{n}}^{T R, \tilde{\sigma}^{n}, X^{n}}\left[Y^{n+1} \Delta B_{n+1}\right] /\left(\tilde{\sigma}^{n}\right)^{2} \Delta t .
$$

\subsection{A useful theorem}

We now present an important theorem that will be useful in our convergence analysis.

Theorem 4.1. Let $\left(X_{t}^{t_{n}, X^{n}}, Y_{t}^{t_{n}, X^{n}}, Z_{t}^{t_{n}, X^{n}}\right)_{t_{n} \leq t \leq T}$ and $\left(X^{n}, Y^{n}, Z^{n}\right)(n=0,1, \ldots, N)$ be the solutions of (2.1)-(2.2) and Scheme 2, respectively. Assume that the functions $f$ and $g$ are Lipschitz continuous with respect to $(x, y, z)$. Then, for sufficiently small $\Delta t$ and $n=$ $0,1, \ldots, N-1$

$$
\begin{aligned}
& \tilde{\mathbb{E}}^{T R}\left[\left|Y_{t_{n}}^{t_{n}, X^{n}}-Y^{n}\right|^{2}+C \Delta t\left|Z_{t_{n}}^{t_{n}, X^{n}}-Z^{n}\right|^{2}\right] \\
& \leq e^{C T} \tilde{\mathbb{E}}^{T R}\left[\left|Y_{t_{N}}^{t_{N}, X^{N}}-Y^{N}\right|^{2}+C \Delta t\left|Z_{t_{N}}^{t_{N}, X^{N}}-Z^{N}\right|^{2}\right] \\
& \quad+C \Delta t \sum_{i=n}^{N-1} \tilde{\mathbb{E}}^{T R}\left[\left|\tilde{Z}^{i}-Z^{i}\right|^{2}\right]+\frac{C}{\Delta t} \sum_{i=n}^{N-1} \tilde{\mathbb{E}}^{T R}\left[\left|\tilde{R}_{y}^{i}\right|^{2}+\left|R_{y}^{G, i}\right|^{2}+\left|\tilde{R}_{z}^{i}\right|^{2}+\left|R_{z}^{G, i}\right|^{2}\right],
\end{aligned}
$$

where $\tilde{Z}^{n}$ is defined in (4.3), $\tilde{R}_{z}^{n}=\tilde{R}_{z}^{n, \sigma^{n}} \vee \tilde{R}_{z}^{n, \tilde{\sigma}^{n}}, R_{z}^{T, n}=R_{z}^{T, n, \sigma^{n}} \vee R_{z}^{T, n, \tilde{\sigma}^{n}}$, and $\tilde{R}_{y}^{n}, R_{y}^{T, n}, \tilde{R}_{z}^{n, \sigma}$, $R_{z}^{T, n, \sigma}$ are defined in (3.8), (3.22), (3.14) and (3.23), respectively.

Proof. For simplicity, we set

$$
\begin{aligned}
& \delta Y_{n}=Y_{t_{n}}^{t_{n}, X^{n}}-Y^{n}, \quad \delta Z_{n}=Z_{t_{n}}^{t_{n}, X^{n}}-Z^{n}, \\
& \delta f_{n}=f\left(t_{n}, X^{n}, Y_{t_{n}}^{t_{n}, X^{n}}, Z_{t_{n}}^{t_{n}, X^{n}}\right)-f\left(t_{n}, X^{n}, Y^{n}, Z^{n}\right), \\
& \delta g_{n}=g\left(t_{n}, X^{n}, Y_{t_{n}}^{t_{n}, X^{n}}, Z_{t_{n}}^{t_{n}, X^{n}}\right)-g\left(t_{n}, X^{n}, Y^{n}, Z^{n}\right) .
\end{aligned}
$$


Step 1. We consider the case of $Y^{n}>Y_{t_{n}}^{t_{n}, X^{n}}$. Subtracting (4.1) from (3.29), we have

$$
\begin{aligned}
Y^{n}-Y_{t_{n}}^{t_{n}, X^{n}}= & \mathbb{E}_{t_{n}}^{T R, \sigma^{n}, X^{n}}\left[Y^{n+1}+f^{n+1} \Delta t+g^{n+1} \Delta\langle B\rangle_{n+1}\right]-\tilde{R}_{y}^{n}-R_{y}^{T, n} \\
& -\mathbb{E}_{t_{n}}^{T R, \tilde{\sigma}^{n}, X^{n}}\left[Y_{t_{n+1}}^{t_{n+1}, X^{n+1}}+f_{t_{n+1}}^{t_{n+1}, X^{n+1}} \Delta t+g_{t_{n+1}}^{t_{n+1}, X^{n+1}} \Delta\langle B\rangle_{n+1}\right] .
\end{aligned}
$$

Noting that

$$
\begin{aligned}
& \mathbb{E}_{t_{n}}^{T R, \sigma^{n}, X^{n}}\left[Y_{t_{n+1}}^{t_{n+1}, X^{n+1}}+f_{t_{n+1}}^{t_{n+1}, X^{n+1}} \Delta t+g_{t_{n+1}}^{t_{n+1}, X^{n+1}} \Delta\langle B\rangle_{n+1}\right] \\
& \leq \mathbb{E}_{t_{n}}^{T R, \tilde{\sigma}^{n}, X^{n}}\left[Y_{t_{n+1}}^{t_{n+1}, X^{n+1}}+f_{t_{n+1}}^{t_{n+1}, X^{n+1}} \Delta t+g_{t_{n+1}}^{t_{n+1}, X^{n+1}} \Delta\langle B\rangle_{n+1}\right],
\end{aligned}
$$

which implies

$$
0<-\delta Y_{n} \leq-\mathbb{E}_{t_{n}}^{T R, \sigma^{n}, X^{n}}\left[\delta Y_{n+1}+\delta f_{n+1} \Delta t+\delta g_{n+1} \Delta\langle B\rangle_{n+1}\right]-\tilde{R}_{y}^{n}-R_{y}^{T, n}
$$

By the Lipschitz continuity of $f, g$ and the definition of $\mathbb{E}_{t_{n}}^{T R, \sigma^{n}, X^{n}}$, we have

$$
\left|\delta Y_{n}\right| \leq\left|\mathbb{E}_{t_{n}}^{T R, \sigma^{n}, X^{n}}\left[\delta Y_{n+1}\right]\right|+C \Delta t \mathbb{E}_{t_{n}}^{T R, \sigma^{n}, X^{n}}\left[\left|\delta Y_{n+1}\right|+\left|\delta Z_{n+1}\right|\right]+\left|\tilde{R}_{y}^{n}\right|+\left|R_{y}^{T, n}\right| .
$$

Taking square on both side of (4.8) and using the inequality $(a+b)^{2} \leq(1+\gamma \Delta t) a^{2}+\left(1+\frac{1}{\gamma \Delta t}\right) b^{2}$, $\gamma>0$, we deduce

$$
\begin{aligned}
\left|\delta Y_{n}\right|^{2} \leq & (1+\gamma \Delta t)\left|\mathbb{E}_{t_{n}}^{T R, \sigma^{n}, X^{n}}\left[\delta Y_{n+1}\right]\right|^{2} \\
& +C\left(1+\frac{1}{\gamma \Delta t}\right)(\Delta t)^{2} \mathbb{E}_{t_{n}}^{T R, \sigma^{n}, X^{n}}\left[\left|\delta Y_{n+1}\right|^{2}+\left|\delta Z_{n+1}\right|^{2}\right] \\
& +C\left(1+\frac{1}{\gamma \Delta t}\right)\left(\left|\tilde{R}_{y}^{n}\right|^{2}+\left|R_{y}^{T, n}\right|^{2}\right) .
\end{aligned}
$$

Let $\sigma=\sigma^{n}$ in (3.25). Combining this with (3.30), we obtain

$$
\left(\sigma^{n}\right)^{2} \delta Z_{n} \Delta t=\mathbb{E}_{t_{n}}^{T R, \sigma^{n}, X^{n}}\left[\delta Y_{n+1} \Delta B_{n+1}\right]+\tilde{R}_{z}^{n, \sigma^{n}}+R_{z}^{T, n, \sigma^{n}}
$$

Taking the square of both sides of (4.10), by Proposition 3.2, we can deduce

$$
\begin{aligned}
\frac{\left(\sigma^{n}\right)^{2}}{C} \Delta t\left|\delta Z_{n}\right|^{2} \leq & \mathbb{E}_{t_{n}}^{T R, \sigma^{n}, X^{n}}\left[\left|\delta Y_{n+1}\right|^{2}\right]-\left|\mathbb{E}_{t_{n}}^{T R, \sigma^{n}, X^{n}}\left[\delta Y_{n+1}\right]\right|^{2} \\
& +\frac{C}{\Delta t}\left(\left|\tilde{R}_{z}^{n, \sigma^{n}}\right|^{2}+\left|R_{z}^{T, n, \sigma^{n}}\right|^{2}\right) .
\end{aligned}
$$


Putting together (4.9) and (4.11), we get

$$
\begin{aligned}
\left|\delta Y_{n}\right|^{2}+\frac{\sigma^{2}}{C} \Delta t\left|\delta Z_{n}\right|^{2} \leq & (1+\gamma \Delta t) \mathbb{E}_{t_{n}}^{T R, \sigma^{n}, X^{n}}\left[\left|\delta Y_{n+1}\right|^{2}\right] \\
& +(1+\gamma \Delta t) \frac{C \Delta t}{\gamma} \mathbb{E}_{t_{n}}^{T R, \sigma^{n}, X^{n}}\left[\left|\delta Y_{n+1}\right|^{2}+\left|\delta Z_{n+1}\right|^{2}\right] \\
& +\frac{C}{\Delta t}\left(\left|\tilde{R}_{y}^{n}\right|^{2}+\left|R_{y}^{T, n}\right|^{2}+\left|\tilde{R}_{z}^{n, \sigma^{n}}\right|^{2}+\left|R_{z}^{T, n, \sigma^{n}}\right|^{2}\right) \\
\leq & (1+C \Delta t) \mathbb{E}_{t_{n}}^{T R, \sigma^{n}, X^{n}}\left[\left|\delta Y_{n+1}\right|^{2}+\frac{\sigma^{2}}{C} \Delta t\left|\delta Z_{n+1}\right|^{2}\right] \\
& +\frac{C}{\Delta t}\left(\left|\tilde{R}_{y}^{n}\right|^{2}+\left|R_{y}^{T, n}\right|^{2}+\left|\tilde{R}_{z}^{n, \sigma^{n}}\right|^{2}+\left|R_{z}^{T, n, \sigma^{n}}\right|^{2}\right)
\end{aligned}
$$

where we have chosen $\gamma=C^{2} / \underline{\sigma}^{2}$. It yields that

$$
\begin{aligned}
\left|\delta Y_{n}\right|^{2}+C \Delta t\left|\delta Z_{n}\right|^{2} \leq & (1+C \Delta t) \mathbb{E}_{t_{n}}^{T R, \sigma^{n}, X^{n}}\left[\left|\delta Y_{n+1}\right|^{2}+C \Delta t\left|\delta Z_{n+1}\right|^{2}\right] \\
& +\frac{C}{\Delta t}\left(\left|\tilde{R}_{y}^{n}\right|^{2}+\left|R_{y}^{T, n}\right|^{2}+\left|\tilde{R}_{z}^{n, \sigma^{n}}\right|^{2}+\left|R_{z}^{T, n, \sigma^{n}}\right|^{2}\right) .
\end{aligned}
$$

Step 2. We consider the case of $Y_{t_{n}}^{t_{n}, X^{n}} \geq Y^{n}$. Subtracting (3.29) from (4.1) and noting that

$$
\begin{aligned}
& \mathbb{E}_{t_{n}}^{T R, \tilde{\sigma}^{n}, X^{n}}\left[Y_{n+1}+f_{n+1} \Delta t+g_{n+1} \Delta\langle B\rangle_{n+1}\right] \\
& \leq \mathbb{E}_{t_{n}}^{T R, \sigma^{n}, X^{n}}\left[Y_{n+1}+f_{n+1} \Delta t+g_{n+1} \Delta\langle B\rangle_{n+1}\right],
\end{aligned}
$$

it follows that

$$
\delta Y_{n} \leq \mathbb{E}_{t_{n}}^{T R, \tilde{\sigma}^{n}, X^{n}}\left[\delta Y_{n+1}+\delta f_{n+1} \Delta t+\delta g_{n+1} \Delta\langle B\rangle_{n+1}\right]+\tilde{R}_{y}^{n}+R_{y}^{T, n}
$$

In addition, by (3.30) and (4.2), we can see

$$
\begin{aligned}
\left(\tilde{\sigma}^{n}\right)^{2} \delta Z_{n} \Delta t & =\left(\tilde{\sigma}^{n}\right)^{2} \Delta t\left(Z_{t_{n}}^{t_{n}, X^{n}}-\tilde{Z}^{n}+\tilde{Z}^{n}-Z^{n}\right) \\
& =\mathbb{E}_{t_{n}}^{T R, \tilde{\sigma}^{n}, X^{n}}\left[\delta Y_{n+1} \Delta B_{n+1}\right]+\left(\tilde{\sigma}^{n}\right)^{2} \Delta t\left(\tilde{Z}^{n}-Z^{n}\right)+\tilde{R}_{z}^{n, \tilde{\sigma}^{n}}+R_{z}^{T, n, \tilde{\sigma}^{n}},
\end{aligned}
$$

where $\tilde{Z}^{n}$ is given in (4.3). Based on (4.14)-(4.15), similar to the step 1 , we can deduce

$$
\begin{aligned}
\left|\delta Y_{n}\right|^{2}+C \Delta t\left|\delta Z_{n}\right|^{2} \leq & (1+C \Delta t) \mathbb{E}_{t_{n}}^{T R, \tilde{\sigma}^{n}, X^{n}}\left[\left|\delta Y_{n+1}\right|^{2}+C \Delta t\left|\delta Z_{n+1}\right|^{2}\right] \\
& +\frac{C}{\Delta t}\left(\left|\tilde{R}_{y}^{n}\right|^{2}+\left|R_{y}^{T, n}\right|^{2}+\left|\tilde{R}_{z}^{n, \tilde{\sigma}^{n}}\right|^{2}+\left|R_{z}^{T, n, \tilde{\sigma}^{n}}\right|^{2}\right) \\
& +C \Delta t\left|\tilde{Z}^{n}-Z^{n}\right|^{2} .
\end{aligned}
$$


Step 3. Together with (4.12) and (4.16), we have

$$
\begin{aligned}
\left|\delta Y_{n}\right|^{2}+C \Delta t\left|\delta Z_{n}\right|^{2} \leq & (1+C \Delta t) \tilde{\mathbb{E}}_{t_{n}}^{T R, X^{n}}\left[\left|\delta Y_{n+1}\right|^{2}+C \Delta t\left|\delta Z_{n+1}\right|^{2}\right] \\
& +\frac{C}{\Delta t}\left(\left|\tilde{R}_{y}^{n}\right|^{2}+\left|R_{y}^{T, n}\right|^{2}+\left|\tilde{R}_{z}^{n}\right|^{2}+\left|R_{z}^{T, n}\right|^{2}\right) \\
& +C \Delta t\left|\tilde{Z}^{n}-Z^{n}\right|^{2}
\end{aligned}
$$

where $\tilde{R}_{z}^{n}=\tilde{R}_{z}^{n, \sigma^{n}} \vee \tilde{R}_{z}^{n, \tilde{\sigma}^{n}}$ and $R_{z}^{T, n}=R_{z}^{T, n, \sigma^{n}} \vee R_{z}^{T, n, \tilde{\sigma}^{n}}$. By Proposition $3.2(a)$, we have

$$
\begin{aligned}
\tilde{\mathbb{E}}^{T R}\left[\left|\delta Y_{n}\right|^{2}+C \Delta t\left|\delta Z_{n}\right|^{2}\right] \leq & (1+C \Delta t) \tilde{\mathbb{E}}^{T R}\left[\left|\delta Y_{n+1}\right|^{2}+C \Delta t\left|\delta Z_{n+1}\right|^{2}\right] \\
& +\frac{C}{\Delta t} \tilde{\mathbb{E}}^{T R}\left[\left|\tilde{R}_{y}^{n}\right|^{2}+\left|R_{y}^{T, n}\right|^{2}+\left|\tilde{R}_{z}^{n}\right|^{2}+\left|R_{z}^{T, n}\right|^{2}\right] \\
& +C \Delta t \tilde{\mathbb{E}}^{T R}\left[\left|\tilde{Z}^{n}-Z^{n}\right|^{2}\right] .
\end{aligned}
$$

Using the induction method, one obtains

$$
\begin{aligned}
\tilde{\mathbb{E}}^{T R}\left[\left|\delta Y_{n}\right|^{2}+C \Delta t\left|\delta Z_{n}\right|^{2}\right] \leq & (1+C \Delta t)^{N-n} \tilde{\mathbb{E}}^{T R}\left[\left|\delta Y_{N}\right|^{2}+C \Delta t\left|\delta Z_{N}\right|^{2}\right] \\
& +\frac{C}{\Delta t} \sum_{i=n}^{N-1}(1+C \Delta t)^{i-n} \tilde{\mathbb{E}}^{T R}\left[\left|\tilde{R}_{y}^{i}\right|^{2}+\left|R_{y}^{T, i}\right|^{2}+\left|\tilde{R}_{z}^{i}\right|^{2}+\left|R_{z}^{T, i}\right|^{2}\right] \\
& +C \Delta t \sum_{i=n}^{N-1}(1+C \Delta t)^{i-n} \tilde{\mathbb{E}}^{T R}\left[\left|\tilde{Z}^{i}-Z^{i}\right|^{2}\right],
\end{aligned}
$$

which yields the result.

\subsection{Error estimates}

To provide the error estimates for Scheme 2, we introduce the following notations. For given constants $\alpha, \beta \in(0,1]$ and $Q=[0, T] \times \mathbb{R}^{m}$, denote

$$
\|u\|_{C^{\alpha, \beta}(Q)}=\sup _{\substack{x, y \in \mathbb{R}^{m}, x \neq y \\ s, t \in[0, T], s \neq t}} \frac{|u(s, x)-u(t, y)|}{|s-t|^{a}+|x-y|^{\beta}},
$$

and

$$
C_{b}^{1+\alpha, 2+\beta}(Q)=\left\{u:\left\|\partial_{t} u\right\|_{C^{\alpha, \beta}(Q)}+\sum_{i=1}^{m}\left\|\partial_{x_{i}} u\right\|_{C^{\alpha, \beta}(Q)}+\sum_{i, j=1}^{m}\left\|\partial_{x_{i} x_{j}}^{2} u\right\|_{C^{\alpha, \beta}(Q)}<\infty\right\} .
$$

Similarly, we can define $C_{b}^{1+\alpha, 2+\beta, 2+\beta, 2+\beta}(Q)$. We need the following assumptions:

(A1) The functions $f(t, x, y, z), g(t, x, y, z), b(t, x), \sigma(t, x)$, and $h(t, x)$ are uniformly Lipschitz continuous with respect to $(x, y, z)$ and Hölder continuous of parameter $\frac{1}{2}$ with respect to $t ; b, \sigma$, and $h$ are bounded; 
(A2) The function $u(t, x) \in C_{b}^{1+1 / 2,2+1}\left([0, T] \times \mathbb{R}^{m}\right)$.

110 Remark 4.2. If $f, g \in C_{b}^{1+1 / 2,2+1,2+1,2+1}\left([0, T] \times \mathbb{R}^{m} \times \mathbb{R} \times \mathbb{R}\right)$ and $\phi \in C_{b}^{2+1}\left(\mathbb{R}^{m}\right)$, by Theorem 6.4.3 in Krylov [22] (see also Theorem 4.4 in Appendix $C$ in Peng [29]), then there exists a constant $\alpha \in(0,1)$ such that for each $\kappa>0, u \in C_{b}^{1+\alpha / 2,2+\alpha}\left([0, T-\kappa] \times \mathbb{R}^{m}\right)$.

We state our convergence theorem here.

Theorem 4.3. Suppose (A1)-(A2) hold. Let $\left(X_{t}^{t_{n}, X^{n}}, Y_{t}^{t_{n}, X^{n}}, Z_{t}^{t_{n}, X^{n}}\right)_{t_{n} \leq t \leq T}$ be the solution of (2.1)-(2.2), and $\left(X^{n}, Y^{n}, Z^{n}\right)(n=0,1, \ldots, N)$ be the solution to Scheme 2 with $Y^{N}=$ $u\left(t_{N}, X^{N}\right)$ and $Z^{N}=D_{x} u\left(t_{N}, X^{N}\right) \sigma\left(t_{N}, X^{N}\right)$. Then, for sufficiently small time step $\Delta t$,

$$
\tilde{\mathbb{E}}^{T R}\left[\left|Y_{t_{n}}^{t_{n}, X^{n}}-Y^{n}\right|^{2}+\Delta t\left|Z_{t_{n}}^{t_{n}, X^{n}}-Z^{n}\right|^{2}\right] \leq C \Delta t
$$

To prove the above theorem, we need the following Lemmas 4.4-4.8.

Lemma 4.4. The numerical scheme (3.27) for $Z^{n}$ is independent of the parameter $\sigma$.

Proof. Notice that

$$
\frac{\mathbb{E}_{t_{n}}^{T R, \sigma, X^{n}}\left[Y^{n+1} \Delta B_{n+1}\right]}{\sigma^{2} \Delta t}=\frac{1}{2 \sqrt{\Delta t}}\left[Y^{n+1}\left(X^{n+1}\left(q_{1}\right)\right) q_{1}+Y^{n+1}\left(X^{n+1}\left(q_{3}\right)\right) q_{3}\right],
$$

where

$$
X^{n+1}\left(q_{i}\right)=X^{n}+b\left(t_{n}, X^{n}\right) \Delta t+h\left(t_{n}, X^{n}\right) \Delta t q_{i}^{2}+\sigma\left(t_{n}, X^{n}\right) \sqrt{\Delta t} q_{i},
$$

and $\left\{q_{i}\right\}_{i=1}^{3}$ is defined in (3.19), which implies the result.

Lemma 4.5. Suppose (A1)-(A2) hold. Let $\hat{R}_{y_{1}}^{n}$ and $\hat{R}_{y_{2}}^{n}$ be the truncation errors defined in (3.5) and (3.6), respectively. Then, for $n=0,1, \ldots, N-1$,

$$
\left|\hat{R}_{y_{1}}^{n}\right| \leq C(\Delta t)^{\frac{3}{2}}, \quad\left|\hat{R}_{y_{2}}^{n}\right| \leq C(\Delta t)^{\frac{3}{2}}
$$

Proof. 1. Recalling the property of $\langle B\rangle$. (see [29], Corollary 3.5.5)

$$
\underline{\sigma}^{2}(t-s) \leq\langle B\rangle_{t}-\langle B\rangle_{s} \leq \bar{\sigma}^{2}(t-s), \text { for } 0 \leq s \leq t<\infty
$$

one has

$$
\begin{aligned}
\left|\hat{R}_{y_{1}}^{n}\right| & \leq \hat{\mathbb{E}}_{t_{n}}^{X^{n}}\left[\left|\int_{t_{n}}^{t_{n+1}}\left(f_{t_{n+1}}^{t_{n}, X^{n}}-f_{t}^{t_{n}, X^{n}}\right) d t+\int_{t_{n}}^{t_{n+1}}\left(g_{t_{n+1}}^{t_{n}, X^{n}}-g_{t}^{t_{n}, X^{n}}\right) d\langle B\rangle_{t}\right|\right] \\
& \leq \hat{\mathbb{E}}_{t_{n}}^{X^{n}}\left[\int_{t_{n}}^{t_{n+1}}\left|f_{t_{n+1}}^{t_{n}, X^{n}}-f_{t}^{t_{n}, X^{n}}\right| d t\right]+C \hat{\mathbb{E}}_{t_{n}}^{X^{n}}\left[\int_{t_{n}}^{t_{n+1}}\left|g_{t_{n+1}}^{t_{n}, X^{n}}-g_{t}^{t_{n}, X^{n}}\right| d t\right] .
\end{aligned}
$$


Under (A1), it is easy to check that

$$
\hat{\mathbb{E}}_{t_{n}}^{X^{n}}\left[\left|X_{t}^{t_{n}, X^{n}}-X_{s}^{t_{n}, X^{n}}\right|^{2}\right] \leq C \Delta t, \text { for } t_{n} \leq s \leq t \leq t_{n+1}
$$

Then, from the Lipschitz continuity of $f, u, D_{x} u$, and $\sigma$, we can derive that

$$
\hat{\mathbb{E}}_{t_{n}}^{X^{n}}\left[\left|f_{t_{n+1}}^{t_{n}, X^{n}}-f_{t}^{t_{n}, X^{n}}\right|^{2}\right] \leq C\left(\left|t_{n+1}-t\right|+\hat{\mathbb{E}}_{t_{n}}^{X^{n}}\left[\left|X_{t_{n+1}}^{t_{n}, X^{n}}-X_{t}^{t_{n}, X^{n}}\right|^{2}\right]\right) \leq C \Delta t
$$

and similarly,

$$
\hat{\mathbb{E}}_{t_{n}}^{X^{n}}\left[\left|g_{t_{n+1}}^{t_{n}, X^{n}}-g_{t}^{t_{n}, X^{n}}\right|^{2}\right] \leq C \Delta t .
$$

Substituting (4.21)-(4.22) into (4.19), by the Cauchy-Schwarz inequality, it is easy to check that $\left|\hat{R}_{y_{1}}^{n}\right| \leq C(\Delta t)^{\frac{3}{2}}$.

2. From the sublinear property of $\hat{\mathbb{E}}_{t_{n}}^{X^{n}}[\cdot]$, we have

$$
\left|\hat{R}_{y_{2}}^{n}\right| \leq \hat{\mathbb{E}}_{t_{n}}^{X^{n}}\left[M_{t_{n+1}}\right] \vee \hat{\mathbb{E}}_{t_{n}}^{X^{n}}\left[-M_{t_{n+1}}\right]
$$

where

$$
M_{t_{n+1}}=Y_{t_{n+1}}^{t_{n}, X^{n}}-Y_{t_{n+1}}^{t_{n+1}, X^{n+1}}+\left(f_{t_{n+1}}^{t_{n}, X^{n}}-f_{t_{n+1}}^{t_{n+1}, X^{n+1}}\right) \Delta t+\left(g_{t_{n+1}}^{t_{n}, X^{n}}-g_{t_{n+1}}^{t_{n+1}, X^{n+1}}\right) \Delta\langle B\rangle_{n+1} .
$$

Next, we only bound the first term on the right-hand side of (4.23), and the second term can be similarly obtained. Define the continuous-time approximation

$$
\tilde{X}_{t}^{t_{n}, X^{n}}=X^{n}+\int_{t_{n}}^{t} b\left(t_{n}, X^{n}\right) d t+\int_{t_{n}}^{t} h\left(t_{n}, X^{n}\right) d\langle B\rangle_{t}+\int_{t_{n}}^{t} \sigma\left(t_{n}, X^{n}\right) d B_{t},
$$

for $t \in\left[t_{n}, t_{n+1}\right]$. Similar to (4.20), we have for $t_{n} \leq s \leq t \leq t_{n+1}$,

$$
\hat{\mathbb{E}}_{t_{n}}^{X^{n}}\left[\left|\tilde{X}_{t}^{t_{n}, X^{n}}-\tilde{X}_{s}^{t_{n}, X^{n}}\right|^{2}\right] \leq C \Delta t
$$

Seeing that $Y_{t_{n+1}}^{t_{n}, X^{n}}=u\left(t_{n+1}, X_{t_{n+1}}^{t_{n}, X^{n}}\right)$ and $Y_{t_{n+1}}^{t_{n+1}, X^{n+1}}=u\left(t_{n+1}, \tilde{X}_{t_{n+1}}^{t_{n}, X^{n}}\right)$, by $G$-Itô's formula and (4.18), we get

$$
\begin{aligned}
& \hat{\mathbb{E}}_{t_{n}}^{X^{n}}\left[Y_{t_{n+1}}^{t_{n}, X^{n}}-Y_{t_{n+1}}^{t_{n+1}, X^{n+1}}\right] \\
& \leq \hat{\mathbb{E}}_{t_{n}}^{X^{n}}\left[\int_{t_{n}}^{t_{n+1}}\left\{L^{0} u\left(t, X_{t}^{t_{n}, X^{n}}\right)-\tilde{L}^{0} u\left(t, \tilde{X}_{t}^{t_{n}, X^{n}}\right)\right\} d t\right] \\
& \quad+\hat{\mathbb{E}}_{t_{n}}^{X^{n}}\left[\int_{t_{n}}^{t_{n+1}}\left\{L^{1} u\left(t, X_{t}^{t_{n}, X^{n}}\right)-\tilde{L}^{1} u\left(t, \tilde{X}_{t}^{t_{n}, X^{n}}\right)\right\} d\langle B\rangle_{t}\right],
\end{aligned}
$$


where

$$
\begin{aligned}
& L^{0}=\partial_{t}+\sum_{i=1}^{m} b_{i}\left(t, X_{t}\right) \partial_{x_{i}}, \quad L^{1}=\sum_{i=1}^{m} h_{i}\left(t, X_{t}\right) \partial_{x_{i}}+\frac{1}{2} \sum_{i, j=1}^{m}\left[\sigma \sigma^{\top}\right]_{i, j}\left(t, X_{t}\right) \partial_{x_{i} x_{j}}^{2}, \\
& \tilde{L}^{0}=\partial_{t}+\sum_{i=1}^{m} b_{i}\left(t_{n}, X^{n}\right) \partial_{x_{i}}, \quad \tilde{L}^{1}=\sum_{i=1}^{m} h_{i}\left(t_{n}, X^{n}\right) \partial_{x_{i}}+\frac{1}{2} \sum_{i, j=1}^{m}\left[\sigma \sigma^{\top}\right]_{i, j}\left(t_{n}, X^{n}\right) \partial_{x_{i} x_{j}}^{2}
\end{aligned}
$$

From the definition of $L^{0}$ and $\tilde{L}^{0}$, we can obtain

$$
\begin{aligned}
& \hat{\mathbb{E}}_{t_{n}}^{X^{n}}\left[\int_{t_{n}}^{t_{n+1}}\left\{L^{0} u\left(t, X_{t}^{t_{n}, X^{n}}\right)-\tilde{L}^{0} u\left(t, \tilde{X}_{t}^{t_{n}, X^{n}}\right)\right\} d t\right] \\
& \leq \hat{\mathbb{E}}_{t_{n}}^{X^{n}}\left[\int_{t_{n}}^{t_{n+1}}\left\{\partial_{t} u\left(t, X_{t}^{t_{n}, X^{n}}\right)-\partial_{t} u\left(t, \tilde{X}_{t}^{t_{n}, X^{n}}\right)\right\} d t\right] \\
& \quad+\hat{\mathbb{E}}_{t_{n}}^{X^{n}}\left[\int_{t_{n}}^{t_{n+1}} D_{x} u\left(t, X_{t}^{t_{n}, X^{n}}\right)\left\{b\left(t, X_{t}^{t_{n}, X^{n}}\right)-b\left(t_{n}, X^{n}\right)\right\} d t\right] \\
& \quad+\hat{\mathbb{E}}_{t_{n}}^{X^{n}}\left[\int_{t_{n}}^{t_{n+1}}\left\{D_{x} u\left(t, X_{t}^{t_{n}, X^{n}}\right)-D_{x} u\left(t, \tilde{X}_{t}^{t_{n}, X^{n}}\right)\right\} b\left(t_{n}, X^{n}\right) d t\right] .
\end{aligned}
$$

Under (A1)-(A2), by (4.20), (4.24) and the Cauchy-Schwarz inequality, we have

$$
\begin{aligned}
& \hat{\mathbb{E}}_{t_{n}}^{X^{n}}\left[\int_{t_{n}}^{t_{n+1}}\left\{L^{0} u\left(t, X_{t}^{t_{n}, X^{n}}\right)-\tilde{L}^{0} u\left(t, \tilde{X}_{t}^{t_{n}, X^{n}}\right)\right\} d t\right] \\
& \leq C \int_{t_{n}}^{t_{n+1}}\left\{\hat{\mathbb{E}}_{t_{n}}^{X^{n}}\left[\left|X_{t}^{t_{n}, X^{n}}-X^{n}\right|\right]+\hat{\mathbb{E}}_{t_{n}}^{X^{n}}\left[\left|\tilde{X}_{t}^{t_{n}, X^{n}}-X^{n}\right|\right]\right\} d t \\
& \leq C(\Delta t)^{\frac{3}{2}} .
\end{aligned}
$$

In the same way as above, we can obtain

$$
\hat{\mathbb{E}}_{t_{n}}^{X^{n}}\left[\int_{t_{n}}^{t_{n+1}}\left\{L^{1} u\left(t, X_{t}^{t_{n}, X^{n}}\right)-\tilde{L}^{1} u\left(t, \tilde{X}_{t}^{t_{n}, X^{n}}\right)\right\} d\langle B\rangle_{t}\right] \leq C(\Delta t)^{\frac{3}{2}} .
$$

Together with (4.25), (4.27), and (4.28), we have

$$
\hat{\mathbb{E}}_{t_{n}}^{X^{n}}\left[Y_{t_{n+1}}^{t_{n}, X^{n}}-Y_{t_{n+1}}^{t_{n+1}, X^{n+1}}\right] \leq C(\Delta t)^{\frac{3}{2}}
$$

Using the Lipschitz condition of $f, g$, and $u$, we can also estimate

$$
\hat{\mathbb{E}}_{t_{n}}^{X^{n}}\left[\left(f_{t_{n+1}}^{t_{n}, X^{n}}-f_{t_{n+1}}^{t_{n+1}, X^{n+1}}\right) \Delta t\right] \leq C(\Delta t)^{\frac{3}{2}}, \quad \hat{\mathbb{E}}_{t_{n}}^{X^{n}}\left[\left(g_{t_{n+1}}^{t_{n}, X^{n}}-g_{t_{n+1}}^{t_{n+1}, X^{n+1}}\right) \Delta\langle B\rangle_{n+1}\right] \leq C(\Delta t)^{\frac{3}{2}} .
$$

Thus our conclusion follows. 
Lemma 4.6. Suppose (A1)-(A2) hold. Let $R_{z_{1}}^{n, \sigma}, R_{z_{2}}^{n, \sigma}$, and $R_{z_{3}}^{n, \sigma}, \sigma \in\{\underline{\sigma}, \bar{\sigma}\}$, be the truncation errors defined in (3.11), (3.12), and (3.15), respectively. Then, for $n=0,1, \ldots, N-1$,

$$
\left|R_{z_{1}}^{n, \sigma}\right| \leq C(\Delta t)^{\frac{3}{2}}, \quad\left|R_{z_{2}}^{n, \sigma}\right| \leq C(\Delta t)^{\frac{3}{2}}, \quad\left|R_{z 3}^{n, \sigma}\right| \leq C(\Delta t)^{\frac{3}{2}} .
$$

Proof. Under (A1)-(A2) and Remark 2.4, the estimates of $R_{z_{1}}^{n, \sigma}, R_{z_{2}}^{n, \sigma}$, and $R_{z 3}^{n, \sigma}$ can be obtained similarly to Lemma 4.5.

For given $\left(t_{n}, X^{n}\right)$, we define the following functions

$$
\begin{aligned}
& \xi_{n}\left(x, y ; X^{n}\right)=X^{n}+b\left(t_{n}, X^{n}\right) \Delta t+\sigma\left(t_{n}, X^{n}\right) x+h\left(t_{n}, X^{n}\right) y, \\
& u_{n}\left(x, y ; X^{n}\right)=u\left(t_{n+1}, \xi_{n}\left(x, y ; X^{n}\right)\right), \\
& v_{n}\left(x, y ; X^{n}\right)=D_{x} u\left(t_{n+1}, \xi_{n}\left(x, y ; X^{n}\right)\right) \sigma\left(t_{n+1}, \xi_{n}\left(x, y ; X^{n}\right)\right), \\
& f_{n}\left(x, y ; X^{n}\right)=f\left(t_{n+1}, \xi_{n}\left(x, y ; X^{n}\right), u_{n}\left(x, y ; X^{n}\right), v_{n}\left(x, y ; X^{n}\right)\right), \\
& g_{n}\left(x, y ; X^{n}\right)=g\left(t_{n+1}, \xi_{n}\left(x, y ; X^{n}\right), u_{n}\left(x, y ; X^{n}\right), v_{n}\left(x, y ; X^{n}\right)\right), \\
& \varphi_{n}\left(x, y ; X^{n}\right)=u_{n}\left(x, y ; X^{n}\right)+f_{n}\left(x, y ; X^{n}\right) \Delta t+g_{n}\left(x, y ; X^{n}\right) y .
\end{aligned}
$$

For convenience, we will omit $X^{n}$ in the following proof, if no ambiguity arises.

Lemma 4.7. Suppose (A1)-(A2) hold. Let $\hat{R}_{y_{3}}^{n}$ be the error defined in (3.9). Then, for $n=0,1, \ldots, N-1$,

$$
\left|\hat{R}_{y_{3}}^{n}\right| \leq C(\Delta t)^{\frac{3}{2}} .
$$

Proof. Noting that

$$
\varphi_{n}\left(\Delta B_{n+1}, \Delta\langle B\rangle_{n+1}\right) \stackrel{d}{=} \varphi_{n}\left(B_{\Delta t},\langle B\rangle_{\Delta t}\right),
$$

in view of (4.29), we can rewrite $\hat{R}_{y_{3}}^{n}$ as

$$
\hat{R}_{y_{3}}^{n}=\hat{\mathbb{E}}_{t_{n}}^{X^{n}}\left[\varphi_{n}\left(B_{\Delta t},\langle B\rangle_{\Delta t}\right)\right]-\tilde{\mathbb{E}}_{t_{n}}^{X^{n}}\left[\varphi_{n}\left(B_{\Delta t},\langle B\rangle_{\Delta t}\right)\right] .
$$

Applying $G$-Itô's formula to $u_{n}\left(B_{\Delta t},\langle B\rangle_{\Delta t}\right)$, we have

$$
\begin{aligned}
u_{n}\left(B_{\Delta t},\langle B\rangle_{\Delta t}\right)= & u_{n}(0,0)+\int_{0}^{\Delta t} \partial_{x} u_{n}\left(B_{s},\langle B\rangle_{s}\right) d B_{s} \\
& +\int_{0}^{\Delta t}\left[\partial_{y} u_{n}\left(B_{s},\langle B\rangle_{s}\right)+\frac{1}{2} \partial_{x x}^{2} u_{n}\left(B_{s},\langle B\rangle_{s}\right)\right] d\langle B\rangle_{s} .
\end{aligned}
$$

Noting that $\hat{\mathbb{E}}\left[\langle B\rangle_{\Delta t}\right]=\bar{\sigma}^{2} \Delta t$ and $-\hat{\mathbb{E}}\left[-\langle B\rangle_{\Delta t}\right]=\underline{\sigma}^{2} \Delta t$, we obtain

$$
\begin{aligned}
& \hat{\mathbb{E}}_{t_{n}}^{X^{n}}\left[\varphi_{n}\left(B B_{\Delta t},\langle B\rangle_{\Delta t}\right)\right] \\
& =\varphi_{n}(0,0)+\hat{\mathbb{E}}_{t_{n}}^{X^{n}}\left[\left\{g_{n}(0,0)+\partial_{y} u_{n}(0,0)+\frac{1}{2} \partial_{x x}^{2} u_{n}(0,0)\right\}\langle B\rangle_{\Delta t}\right]+\mathcal{E}_{n} \\
& =\varphi_{n}(0,0)+G\left(2 g_{n}(0,0)+2 \partial_{y} u_{n}(0,0)+\partial_{x x}^{2} u_{n}(0,0)\right) \Delta t+\mathcal{E}_{n},
\end{aligned}
$$


where

$$
\begin{aligned}
\mathcal{E}_{n}= & \hat{\mathbb{E}}_{t_{n}}^{X^{n}}\left[\int_{0}^{\Delta t}\left\{\partial_{y} u_{n}\left(B_{s},\langle B\rangle_{s}\right)+\frac{1}{2} \partial_{x x}^{2} u_{n}\left(B_{s},\langle B\rangle_{s}\right)\right\} d\langle B\rangle_{s}\right. \\
& \left.+g_{n}\left(B_{\Delta t},\langle B\rangle_{\Delta t}\right)\langle B\rangle_{\Delta t}+f_{n}\left(B_{\Delta t},\langle B\rangle_{\Delta t}\right) \Delta t\right]-f_{n}(0,0) \Delta t \\
& -\hat{\mathbb{E}}_{t_{n}}^{X^{n}}\left[\left\{g_{n}(0,0)+\partial_{y} u_{n}(0,0)+\frac{1}{2} \partial_{x x}^{2} u_{n}(0,0)\right\}\langle B\rangle_{\Delta t}\right] .
\end{aligned}
$$

With the help of (4.18), one can check that

$$
\begin{aligned}
\left|\mathcal{E}_{n}\right| \leq & C \hat{\mathbb{E}}_{t_{n}}^{X^{n}}\left[\int_{0}^{\Delta t}\left|f_{n}\left(B_{\Delta t},\langle B\rangle_{\Delta t}\right)-f_{n}(0,0)\right|+\left|g_{n}\left(B_{\Delta t},\langle B\rangle_{\Delta t}\right)-g_{n}(0,0)\right| d s\right] \\
& +C \hat{\mathbb{E}}_{t_{n}}^{X^{n}}\left[\int_{0}^{\Delta t}\left|\partial_{y} u_{n}\left(B_{s},\langle B\rangle_{s}\right)-\partial_{y} u_{n}(0,0)\right| d s\right] \\
& +C \hat{\mathbb{E}}_{t_{n}}^{X^{n}}\left[\int_{0}^{\Delta t}\left|\partial_{x x}^{2} u_{n}\left(B_{s},\langle B\rangle_{s}\right)-\partial_{x x}^{2} u_{n}(0,0)\right| d s\right] .
\end{aligned}
$$

In view of the assumptions (A1)-(A2), we get $\left|\mathcal{E}_{n}\right| \leq C(\Delta t)^{3 / 2}$. Thus

$$
\begin{aligned}
& \hat{\mathbb{E}}_{t_{n}}^{X^{n}}\left[\varphi_{n}\left(B \Delta t,\langle B\rangle_{\Delta t}\right)\right] \\
& =\varphi_{n}(0,0)+G\left(2 g_{n}(0,0)+2 \partial_{y} u_{n}(0,0)+\partial_{x x}^{2} u_{n}(0,0)\right) \Delta t+\mathcal{O}(\Delta t)^{\frac{3}{2}} .
\end{aligned}
$$

Similarly, we have

$$
\begin{aligned}
& \tilde{\mathbb{E}}_{t_{n}}^{X^{n}}\left[\varphi_{n}\left(B \Delta t,\langle B\rangle_{\Delta t}\right)\right] \\
& =\varphi_{n}(0,0)+\sup _{\sigma \in\{\underline{\sigma}, \bar{\sigma}\}} \mathbb{E}_{t_{n}}^{\sigma, X^{n}}\left[\left\{g_{n}(0,0)+\partial_{y} u_{n}(0,0)+\frac{1}{2} \partial_{x x}^{2} u_{n}(0,0)\right\}\langle B\rangle_{\Delta t}\right]+\tilde{\mathcal{E}}_{n} \\
& =\varphi_{n}(0,0)+G\left(2 g_{n}(0,0)+2 \partial_{y} u_{n}(0,0)+\partial_{x x}^{2} u_{n}(0,0)\right) \Delta t+\tilde{\mathcal{E}}_{n},
\end{aligned}
$$

where

$$
\begin{aligned}
\tilde{\mathcal{E}}_{n}= & \tilde{\mathbb{E}}_{t_{n}}^{X^{n}}\left[\int_{0}^{\Delta t}\left\{\partial_{y} u_{n}\left(B_{s},\langle B\rangle_{s}\right)+\frac{1}{2} \partial_{x x}^{2} u_{n}\left(B_{s},\langle B\rangle_{s}\right)\right\} d\langle B\rangle_{s}\right. \\
& \left.+g_{n}\left(B_{\Delta t},\langle B\rangle_{\Delta t}\right)\langle B\rangle_{\Delta t}+f_{n}\left(B_{\Delta t},\langle B\rangle_{\Delta t}\right) \Delta t\right]-f_{n}(0,0) \Delta t \\
& -\tilde{\mathbb{E}}_{t_{n}}^{X^{n}}\left[\left\{g_{n}(0,0)+\partial_{y} u_{n}(0,0)+\frac{1}{2} \partial_{x x}^{2} u_{n}(0,0)\right\}\langle B\rangle_{\Delta t}\right] .
\end{aligned}
$$

By a simple calculation similar to $\mathcal{E}_{n}$, we have $\left|\tilde{\mathcal{E}}_{n}\right| \leq C(\Delta t)^{3 / 2}$. Together with (4.30), (4.33) and (4.34), the desired result follows. 
Lemma 4.8. Suppose (A1)-(A2) hold. Let $R_{y}^{T, n}$ and $R_{z}^{T, n, \sigma}, \sigma \in\{\underline{\sigma}, \bar{\sigma}\}$ be the errors defined in (3.22) and (3.23), respectively. Then for $n=0,1, \ldots, N-1$,

$$
\left|R_{y}^{T, n}\right| \leq C(\Delta t)^{\frac{3}{2}}, \quad\left|R_{z}^{T, n, \sigma}\right| \leq C(\Delta t)^{\frac{3}{2}}
$$

Proof. From (4.29), it is easy to know

$$
R_{y}^{T, n}=\tilde{\mathbb{E}}_{t_{n}}^{X^{n}}\left[\varphi_{n}\left(B_{\Delta t},\langle B\rangle_{\Delta t}\right)\right]-\tilde{\mathbb{E}}_{t_{n}}^{T R, X^{n}}\left[\varphi_{n}\left(B_{\Delta t},\langle B\rangle_{\Delta t}\right)\right]
$$

Under the assumptions (A1)-(A2), applying the Taylor expansion to $u_{n}$ and using the Lipschitz condition on $f_{n}$ and $g_{n}$, we get

$$
\begin{aligned}
\varphi_{n}\left(\sqrt{\Delta t} q_{i}, \Delta t q_{i}^{2}\right)= & u_{n}(0,0)+\partial_{x} u_{n}(0,0) \sqrt{\Delta t} q_{i}+\partial_{y} u_{n}(0,0) \Delta t q_{i}^{2} \\
& +\frac{1}{2} \partial_{x x}^{2} u_{n}(0,0)\left(\sqrt{\Delta t} q_{i}\right)^{2}+f_{n}(0,0) \Delta t \\
& +g_{n}(0,0) \Delta t q_{i}^{2}+\mathcal{O}(\Delta t)^{\frac{3}{2}}
\end{aligned}
$$

This and Remark 3.1 yield

$$
\begin{aligned}
& \tilde{\mathbb{E}}_{t_{n}}^{T R, X^{n}}\left[\varphi_{n}\left(B_{\Delta t},\langle B\rangle_{\Delta t}\right)\right] \\
& =\varphi_{n}(0,0)+\sup _{\sigma \in\{\underline{\sigma}, \bar{\sigma}\}}\left[\left\{g_{n}(0,0)+\partial_{y} u_{n}(0,0)+\frac{1}{2} \partial_{x x}^{2} u_{n}(0,0)\right\} \sigma^{2} \Delta t\right]+\mathcal{O}(\Delta t)^{\frac{3}{2}} \\
& =\varphi_{n}(0,0)+G\left(2 g_{n}(0,0)+2 \partial_{y} u_{n}(0,0)+\partial_{x x}^{2} u_{n}(0,0)\right) \Delta t+\mathcal{O}(\Delta t)^{\frac{3}{2}} .
\end{aligned}
$$

125 Together with (4.34)-(4.35) and (4.37), we obtain $\left|R_{y}^{T, n}\right| \leq C(\Delta t)^{\frac{3}{2}}$. Similarly, according to (3.7) and (3.18), the estimate for $R_{z}^{T, n, \sigma}$ follows.

Proof of Theorem 4.3. From Lemmas 4.5-4.8, it holds that

$$
\begin{aligned}
& \left|\tilde{R}_{y}^{n}\right|^{2} \leq C(\Delta t)^{3}, \quad\left|R_{y}^{T, n}\right|^{2} \leq C(\Delta t)^{3} \\
& \left|\tilde{R}_{z}^{n}\right|^{2} \leq C(\Delta t)^{3}, \quad\left|R_{z}^{T, n}\right|^{2} \leq C(\Delta t)^{3}
\end{aligned}
$$

Then by Theorem 4.1 and Lemma 4.4, the conclusion can be obtained.

Remark 4.9. Suppose that $f, g \in C_{b}^{1+1 / 2,2+1,2+1,2+1}\left([0, T] \times \mathbb{R}^{m} \times \mathbb{R} \times \mathbb{R}\right)$ and $\phi \in C_{b}^{2+1}\left(\mathbb{R}^{m}\right)$. Without (A2), we conclude that

$$
\tilde{\mathbb{E}}^{T R}\left[\left|Y_{t_{n}}^{t_{n}, X^{n}}-Y^{n}\right|^{2}\right] \leq C(\Delta t)^{\alpha}, \text { for some } \alpha \in(0,1),
$$

which yields Scheme 2 is of order $\alpha / 2$ for $Y$. 


\section{Multi-dimensional $G$-Brownian motion case}

In this section, we extend our results to the multi-dimensional $G$-Brownian motion case. Let $G: \mathbb{S}(d) \rightarrow \mathbb{R}$ be a given sublinear function such that

$$
G(A)=\frac{1}{2} \sup _{Q \in \Sigma} \operatorname{tr}[Q A]
$$

with the closed set $\Sigma \subset \mathbb{S}^{+}(d)$, and $B_{t}=\left(B_{t}^{1}, \ldots, B_{t}^{d}\right)^{\top}$ be the corresponding $d$-dimensional $G$-Brownian motion. Denote $h(t, x):=\left(h_{i j}(t, x)\right)_{i, j=1}^{d}$ and $g(t, x, y, z):=\left(g_{i j}(t, x, y, z)\right)_{i, j=1}^{d}$. For $\eta \in L_{G}^{1}\left(\Omega_{T}\right)$, define the approximate conditional $G$-expectation $\tilde{\mathbb{E}}_{t_{n}}^{X^{n}}[\eta]$ by

$$
\tilde{\mathbb{E}}_{t_{n}}^{X^{n}}[\eta]=\sup _{Q \in \Sigma} \mathbb{E}_{t_{n}}^{Q, X^{n}}[\eta]=\sup _{Q \in \Sigma} \mathbb{E}_{P^{Q}}\left[\eta \mid X_{t_{n}}=X^{n}\right]
$$

where $P^{Q} \in \mathcal{P}$ is a probability measure, under which $\left(B_{t}\right)_{t \geq 0}$ is the classical Brownian motion with $\mathbb{E}_{P^{Q}}\left[B_{t}\right]=0$ and $\mathbb{E}_{P^{Q}}\left[B_{t} B_{t}^{\top}\right]=Q t$. Then the equations (3.16)-(3.17) can be extended to

$$
\begin{aligned}
& Y_{t_{n}}^{t_{n}, X^{n}}=\tilde{\mathbb{E}}_{t_{n}}^{X^{n}}\left[Y_{t_{n+1}}^{t_{n+1}, X^{n+1}}+f_{t_{n+1}}^{t_{n+1}, X^{n+1}} \Delta t+\left\langle g_{t_{n+1}}^{t_{n+1}, X^{n+1}}, \Delta\langle B\rangle_{n+1}\right\rangle\right]+\tilde{R}_{y}^{n}, \\
& Z_{t_{n}}^{t_{n}, X^{n}} Q \Delta t=\mathbb{E}_{t_{n}}^{Q, X^{n}}\left[Y_{t_{n+1}}^{t_{n+1}, X^{n+1}} \Delta B_{n+1}^{\top}\right]+\tilde{R}_{z}^{n, Q}, \text { for } Q \in \Sigma,
\end{aligned}
$$

where $Z_{t}=\left(Z_{t}^{1}, \ldots, Z_{t}^{d}\right), \tilde{R}_{y}^{n}$ is given in (3.8) and $\tilde{R}_{z}^{n, Q}=R_{z_{1}}^{n, Q}+R_{z_{2}}^{n, Q}-R_{z_{3}}^{n, Q}$ with

$$
\begin{aligned}
R_{z_{1}}^{n, Q}= & \mathbb{E}_{t_{n}}^{Q, X^{n}}\left[\int_{t_{n}}^{t_{n+1}} f_{t}^{t_{n}, X^{n}} d t \Delta B_{n+1}^{\top}+\int_{t_{n}}^{t_{n+1}} g_{t}^{t_{n}, X^{n}} d\langle B\rangle_{t} \Delta B_{n+1}^{\top}\right] \\
& -\mathbb{E}_{t_{n}}^{Q, X^{n}}\left[\left(K_{t_{n+1}}^{t_{n}, X^{n}}-K_{t_{n}}^{t_{n}, X^{n}}\right) \Delta B_{n+1}^{\top}\right], \\
R_{z_{2}}^{n, Q}= & \mathbb{E}_{t_{n}}^{Q, X^{n}}\left[Y_{t_{n+1}}^{t_{n}, X^{n}} \Delta B_{n+1}^{\top}\right]-\mathbb{E}_{t_{n}}^{Q, X^{n}}\left[Y_{t_{n+1}}^{t_{n+1}, X^{n+1}} \Delta B_{n+1}^{\top}\right], \\
R_{z_{3}}^{n, Q}= & \mathbb{E}_{t_{n}}^{Q, X^{n}}\left[\int_{t_{n}}^{t_{n+1}} Z_{t}^{t_{n}, X^{n}} Q d t\right]-Z_{t_{n}}^{t_{n}, X^{n}} Q \Delta t .
\end{aligned}
$$

Example 5.1. Assume that $d=2$ and $Q=\operatorname{diag}\left(\sigma_{1}^{2}, \sigma_{2}^{2}\right)$. In this case, we have

$$
\mathbb{E}_{t_{n}}^{Q, X^{n}}\left[\varphi\left(\Delta B_{n+1}^{1}, \Delta B_{n+1}^{2}\right) \Delta B_{n+1}^{1}\right]=\mathbb{E}_{t_{n}}^{\sigma_{1}, X^{n}}\left[\mathbb{E}_{t_{n}}^{\sigma_{2}, X^{n}}\left[\varphi\left(x, \Delta B_{n+1}^{2}\right) x\right]_{x=\Delta B_{n+1}^{1}}\right]
$$

From the trinomial tree rule (3.18), we can deduce that

$$
\begin{aligned}
\frac{\mathbb{E}_{t_{n}}^{T R, Q, X^{n}}\left[\varphi\left(\Delta B_{n+1}\right) \Delta B_{n+1}^{1}\right]}{\sigma_{1}^{2} \Delta t} & =\frac{1}{\sigma_{1}^{2} \sqrt{\Delta t}} \sum_{i=1}^{3} \sum_{j=1}^{3} \omega_{i}^{\sigma_{1}} \omega_{j}^{\sigma_{2}} \varphi\left(\sqrt{\Delta t} q_{i}, \sqrt{\Delta t} q_{j}\right) q_{i} \\
& =\frac{1}{2 \sqrt{\Delta t}} \sum_{j=1}^{3} \omega_{j}^{\sigma_{2}}\left[\varphi\left(\sqrt{\Delta t}, \sqrt{\Delta t} q_{j}\right)-\varphi\left(-\sqrt{\Delta t}, \sqrt{\Delta t} q_{j}\right)\right]
\end{aligned}
$$


which is independent of $\sigma_{1}$ but dependent on $\sigma_{2}$. Similarly,

$$
\frac{\mathbb{E}_{t_{n}}^{T R, Q, X^{n}}\left[\varphi\left(\Delta B_{n+1}\right) \Delta B_{n+1}^{2}\right]}{\sigma_{2}^{2} \Delta t}=\frac{1}{2 \sqrt{\Delta t}} \sum_{i=1}^{3} \omega_{i}^{\sigma_{1}}\left[\varphi\left(\sqrt{\Delta t} q_{i}, \sqrt{\Delta t}\right)-\varphi\left(\sqrt{\Delta t} q_{i},-\sqrt{\Delta t}\right)\right],
$$

which is independent of $\sigma_{2}$ but dependent on $\sigma_{1}$.

Remark 5.2. Example 5.1 implies that the trinomial tree rule (3.27) for $Z^{n}$ depends on $Q$ in the case of multi-dimensional G-Brownian motion, and Lemma 4.4 is no longer true. In addition, in this case the weights of the trinomial tree rule are determined by the matrix $Q \in \Sigma$, which makes it more difficult to derive.

\subsection{Gauss-Hermite quadrature rule}

Inspired by the approximation of classical $d$-dimensional Brownian motion distribution, for a function $\varphi: \mathbb{R}^{m} \times \mathbb{R}^{d} \times \mathbb{R}^{d \times d} \rightarrow \mathbb{R}$, denote $\varphi_{t_{n+1}}=\varphi\left(X^{n+1}, \Delta B_{n+1}, \Delta\langle B\rangle_{n+1}\right)$, we introduce a more effective Gauss-Hermite quadrature rule to approximate $\tilde{\mathbb{E}}_{t_{n}}^{G H, X^{n}}\left[\varphi_{t_{n+1}}\right]$ as follows:

$$
\begin{aligned}
\tilde{\mathbb{E}}_{t_{n}}^{G H, X^{n}}\left[\varphi_{t_{n+1}}\right] & :=\sup _{Q \in \Sigma} \mathbb{E}_{t_{n}}^{G H, Q, X^{n}}\left[\varphi\left(X^{n+1}, \Delta B_{n+1}, \Delta\langle B\rangle_{n+1}\right)\right] \\
& :=\sup _{Q \in \Sigma} \sum_{i_{1}, \ldots, i_{d}=1}^{L} \omega_{i_{1}} \cdots \omega_{i_{d}} \tilde{\varphi}\left(X^{n}, \sqrt{\Delta t} P_{Q}, \Delta t Q\right),
\end{aligned}
$$

where $\tilde{\varphi}(x, y, z)=\varphi\left(x+b\left(t_{n}, x\right) \Delta t+\sigma\left(t_{n}, x\right) y+\left\langle h\left(t_{n}, x\right), z\right\rangle, y, z\right)$, the weights $\omega_{i}=\frac{2^{L+1} L !}{\left[H_{L}^{\prime}\left(x_{i}\right)\right]^{2}}$ for $i=1, \ldots L, P_{Q}=\sqrt{Q}\left(\sqrt{2} p_{i_{1}}, \ldots, \sqrt{2} p_{i_{d}}\right)^{\top}$, and the nodes $\left\{p_{i}\right\}_{i=1}^{L}$ are the roots of the $L$ degree Hermite polynomial $H_{L}(x)=(-1)^{L} e^{x^{2}} \frac{d^{L}}{d x^{L}} e^{-x^{2}}$. Similarly, we define the associated discrete sublinear expectation

$$
\tilde{\mathbb{E}}^{G H}\left[\varphi_{t_{n+1}}\right]=\tilde{\mathbb{E}}_{t_{0}}^{G H, x_{0}}\left[\tilde{\mathbb{E}}_{t_{1}}^{G H, X^{1}}\left[\cdots \tilde{\mathbb{E}}_{t_{n}}^{G H, X^{n}}\left[\varphi_{t_{n+1}}\right]\right]\right] .
$$

Remark 5.3. For any fixed $L \geq 2$, noting that the Gauss-Hermite quadrature rule is exact for any polynomial of degree $\leq 2 L-1$, one can check that

$$
\sum_{i=1}^{L} \omega_{i}=1, \quad \sum_{i=1}^{L} \omega_{i} p_{i}=0, \quad \sum_{i=1}^{L} \omega_{i} p_{i}^{2}=\frac{1}{2}
$$

Similar to the one-dimensional case, based on (5.3) and (5.4), we get the following reference equations:

$$
\begin{aligned}
& Y_{t_{n}}^{t_{n}, X^{n}}=\tilde{\mathbb{E}}_{t_{n}}^{G H, X^{n}}\left[Y_{t_{n+1}}^{t_{n+1}, X^{n+1}}+f_{t_{n+1}}^{t_{n+1}, X^{n+1}} \Delta t+\left\langle g_{t_{n+1}}^{t_{n+1}, X^{n+1}}, \Delta\langle B\rangle_{n+1}\right\rangle\right]+\tilde{R}_{y}^{n}+R_{y}^{G, n}, \\
& Z_{t_{n}}^{t_{n}, X^{n}} Q \Delta t=\mathbb{E}_{t_{n}}^{G H, Q, X^{n}}\left[Y_{t_{n+1}}^{t_{n+1}, X^{n+1}} \Delta B_{n+1}^{\top}\right]+\tilde{R}_{z}^{n, Q}+R_{z}^{G, n, Q}, \text { for } Q \in \Sigma
\end{aligned}
$$


where

$$
\begin{aligned}
R_{y}^{G, n}= & \tilde{\mathbb{E}}_{t_{n}}^{X^{n}}\left[Y_{t_{n+1}}^{t_{n+1}, X^{n+1}}+f_{t_{n+1}}^{t_{n+1}, X^{n+1}} \Delta t+\left\langle g_{t_{n+1}}^{t_{n+1}, X^{n+1}}, \Delta\langle B\rangle_{n+1}\right\rangle\right] \\
& -\tilde{\mathbb{E}}_{t_{n}}^{G H, X^{n}}\left[Y_{t_{n+1}}^{t_{n+1}, X^{n+1}}+f_{t_{n+1}}^{t_{n+1}, X^{n+1}} \Delta t+\left\langle g_{t_{n+1}}^{t_{n+1}, X^{n+1}}, \Delta\langle B\rangle_{n+1}\right\rangle\right], \\
R_{z}^{G, n, Q}= & \mathbb{E}_{t_{n}}^{Q, X^{n}}\left[Y_{t_{n+1}}^{t_{n+1}, X^{n+1}} \Delta B_{n+1}^{\top}\right]-\mathbb{E}_{t_{n}}^{G H, Q, X^{n}}\left[Y_{t_{n+1}}^{t_{n+1}, X^{n+1}} \Delta B_{n+1}^{\top}\right] .
\end{aligned}
$$

\subsection{Numerical schemes and convergence results}

Let $Y^{n}$ and $Z^{n}$ be the approximate values of the solutions $Y_{t}$ and $Z_{t}$ of the $G$-FBSDE (1.1) at time $t_{n}$, respectively, and denote $f^{n+1}=f\left(t_{n+1}, X^{n+1}, Y^{n+1}, Z^{n+1}\right), g^{n+1}=g\left(t_{n+1}, X^{n+1}\right.$, $\left.Y^{n+1}, Z^{n+1}\right)$. By removing the error terms $\tilde{R}_{y}^{n}, R_{y}^{G, n}, \tilde{R}_{z}^{n, Q}$, and $R_{z}^{G, n, Q}$ from (5.10)-(5.11), we obtain our discrete scheme for solving the G-FBSDE (1.1) as follows.

Scheme 3. Given random variables $Y^{N}$ and $Z^{N}$, for $n=N-1, \ldots, 0$ and $Q \in \Sigma$, solve random variables $Y^{n}=Y^{n}\left(X^{n}\right)$ and $Z^{n}=Z^{n}\left(X^{n}\right)$ from

$$
\begin{aligned}
Y^{n} & =\tilde{\mathbb{E}}_{t_{n}}^{G H, X^{n}}\left[Y^{n+1}+f^{n+1} \Delta t+\left\langle g^{n+1}, \Delta\langle B\rangle_{n+1}\right\rangle\right], \\
Z^{n} & =\mathbb{E}_{t_{n}}^{G H, Q, X^{n}}\left[Y^{n+1} \Delta B_{n+1}^{\top}\right] Q^{-1} / \Delta t,
\end{aligned}
$$

with

$$
X^{n+1}=X^{n}+b\left(t_{n}, X^{n}\right) \Delta t+\left\langle h\left(t_{n}, X^{n}\right), \Delta\langle B\rangle_{n+1}\right\rangle+\sigma\left(t_{n}, X^{n}\right) \Delta B_{n+1} .
$$

Compared with the trinomial tree scheme in the one-dimensional G-Brownian motion case, (5.13) in Scheme 3 for $Z^{n}$ depends on the parameter $Q$ in the multi-dimensional case. To obtain the convergence results for Scheme 3, the following assumption need to be imposed. (A3) There exists a constant $C>0$, such that, for any $\tilde{Q} \in \Sigma$,

$$
\sup _{0 \leq n \leq N-1}\left|Z^{n}-\tilde{Z}^{n}\right| \leq C(\Delta t)^{\frac{1}{2}}
$$

where

$$
\tilde{Z}^{n}=\mathbb{E}_{t_{n}}^{G H, \tilde{Q}, X^{n}}\left[Y^{n+1} \Delta B_{n+1}\right] \tilde{Q}^{-1} / \Delta t .
$$

Let us give a sufficient condition for the assumption (A3) to illustrate its rationality.

Proposition 5.4. For each fixed $\left(t_{n}, X^{n}\right)$, assume that there exists a function $\psi_{n} \in C^{1}\left(\mathbb{R}^{d} \times\right.$ $\left.\mathbb{R}^{d \times d} ; \mathbb{R}\right)$ such that $Y^{n+1}=Y^{n+1}\left(X^{n+1}\right)=\psi_{n}\left(\Delta B_{n+1}, \Delta\langle B\rangle_{n+1}\right)$, and for any $x, x^{\prime} \in \mathbb{R}^{d}$, $y, y^{\prime} \in \mathbb{R}^{d \times d}$,

$$
\begin{aligned}
& \sup _{0 \leq n \leq N-1}\left|D_{x} \psi_{n}(x, y)-D_{x} \psi_{n}\left(x^{\prime}, y^{\prime}\right)\right| \leq C\left(\left|x-x^{\prime}\right|+\left|y-y^{\prime}\right|\right), \\
& \sup _{0 \leq n \leq N-1}\left|D_{y} \psi_{n}(x, y)-D_{y} \psi_{n}\left(x^{\prime}, y^{\prime}\right)\right| \leq C\left(\left|x-x^{\prime}\right|+\left|y-y^{\prime}\right|\right) .
\end{aligned}
$$

Then, the assumption (A3) holds. 
Proof. Notice that

$$
\mathbb{E}_{t_{n}}^{G H, Q, X^{n}}\left[Y^{n+1} \Delta B_{n+1}^{\top}\right]=\sum_{i_{1}, \ldots, i_{d}=1}^{L} \omega_{i_{1}} \cdots \omega_{i_{d}} \psi_{n}\left(\sqrt{\Delta t} P_{Q}, \Delta t Q\right) \sqrt{\Delta t} P_{Q}^{\top} .
$$

Using the Taylor expansion and (5.16), for $\theta \in(0,1)$, we have

$$
\begin{aligned}
\psi_{n}\left(\sqrt{\Delta t} P_{Q}, \Delta t Q\right)= & \psi_{n}(0,0)+D_{x} \psi_{n}(0,0) P_{Q} \sqrt{\Delta t}+\left\langle D_{y} \psi_{n}(0,0), Q\right\rangle \Delta t \\
& +\int_{0}^{1}\left[D_{x} \psi_{n}\left(\theta \sqrt{\Delta t} P_{Q}, \theta \Delta t Q\right)-D_{x} \psi_{n}(0,0)\right] d \theta P_{Q} \sqrt{\Delta t} \\
& +\int_{0}^{1}\left\langle D_{y} \psi_{n}\left(\theta \sqrt{\Delta t} P_{Q}, \theta \Delta t Q\right)-D_{y} \psi_{n}(0,0), Q\right\rangle d \theta \Delta t \\
= & \psi_{n}(0,0)+D_{x} \psi_{n}(0,0) P_{Q} \sqrt{\Delta t}+\mathcal{O}(\Delta t) .
\end{aligned}
$$

From (5.17)-(5.18), one can check that

$$
Z^{n}=D_{x} \psi_{n}(0,0)+\mathcal{O}(\Delta t)^{1 / 2}
$$

Analogously,

$$
\tilde{Z}^{n}=D_{x} \psi_{n}(0,0)+\mathcal{O}(\Delta t)^{1 / 2} .
$$

The desired result follows.

Similar to the analysis in Theorems 4.1 and 4.3, under the assumptions (A1)-(A3), we derive the following convergence theorem.

Theorem 5.5. Suppose (A1)-(A3) hold. Let $\left(X_{t}^{t_{n}, X^{n}}, Y_{t}^{t_{n}, X^{n}}, Z_{t}^{t_{n}, X^{n}}\right)_{t_{n} \leq t \leq T}$ be the solution of (2.1)-(2.2), and $\left(X^{n}, Y^{n}, Z^{n}\right)(n=0,1, \ldots, N)$ be the solution to Scheme 3 with $Y^{N}=$ $u\left(t_{N}, X^{N}\right)$ and $Z^{N}=D_{x} u\left(t_{N}, X^{N}\right) \sigma\left(t_{N}, X^{N}\right)$. Then, for sufficiently small time step $\Delta t$,

$$
\tilde{\mathbb{E}}^{G H}\left[\left|Y_{t_{n}}^{t_{n}, X^{n}}-Y^{n}\right|^{2}+\Delta t\left|Z_{t_{n}}^{t_{n}, X^{n}}-Z^{n}\right|^{2}\right] \leq C \Delta t .
$$

Remark 5.6. In the case when $f=g=0$, without the assumption (A3), we have

$$
\tilde{\mathbb{E}}^{G H}\left[\left|Y_{t_{n}}^{t_{n}, X^{n}}-Y^{n}\right|^{2}\right] \leq C \Delta t,
$$

which indicates that Scheme 3 is of order $1 / 2$ for solving the G-heat equation introduced by Peng [29]

$$
\left\{\begin{array}{l}
\partial_{t} u+G\left(D_{x x}^{2} u\right)=0, \quad \forall(t, x) \in(0, T] \times \mathbb{R}^{m} \\
u(T, x)=\phi(x)
\end{array}\right.
$$




\section{Numerical experiments}

In this section, some numerical experiments will be carried out to illustrate the high accuracy of our numerical schemes. We take a uniform partition with the time step $\Delta t=\frac{T}{N}$ and introduce the uniform space partition $\mathcal{D}_{h}=\mathcal{D}_{1, h} \times \mathcal{D}_{2, h} \times \cdots \times \mathcal{D}_{m, h}$, where $\mathcal{D}_{j, h}$ is the partition of the one-dimensional real axis $\mathbb{R}$

$$
\mathcal{D}_{j, h}=\left\{x_{k}^{j} \mid x_{k}^{j}=k \Delta x, k=0, \pm 1, \pm 2, \ldots\right\},
$$

for $j=1,2, \ldots, m$, and $\Delta x$ is a suitable spatial step. Let $\left|Y_{0}-Y^{0}\right|$ and $\left|Z_{0}-Z^{0}\right|$ represent the errors between the exact and numerical solutions for $Y$ and $Z$ at $\left(t_{0}, x_{0}\right)$, and denote $\varphi(x)=x \wedge 1 \vee(-1)$. In our tables, we also denote by CR the convergence rate, TR the discrete Scheme 2, and GH the discrete Scheme 3, respectively. When it comes to Scheme 3, in order to avoid confusion, we assume that $\widetilde{\mathbb{E}}_{t_{n}}^{G H, X^{n}}[\cdot]$ reaches its maximum at $\mathbb{E}_{t_{n}}^{G H, Q_{y}^{n}, X^{n}}[\cdot]$ with the parameter $Q_{y}^{n}$, and use the notation $Q_{z}^{n}$ to further distinguish the selected parameter for solving $Z$.

\subsection{G-heat equation}

We first apply our schemes to the $G$-heat equation (5.21) which is related to the $G$-FBSDE (2.1)-(2.2) with $f=g=0$.

Example 6.1. Consider the G-heat equation (5.21) with the terminal $\varphi(x)=\left(x+c_{1}\right)^{3}$. We set $T=1, x_{0}=0, \underline{\sigma}=0.2, \bar{\sigma}=1$, and $c_{1}=-0.5840$. The solution of this $G$-heat equation given in [18] is $u(t, x)=(1-t)^{\frac{3}{2}} P\left(\frac{x+c_{1}}{\sqrt{1-t}}\right)$, where

$$
P(x)= \begin{cases}3 x+x^{3}+\frac{k_{1}}{2}\left[\left(2+x^{2}\right) e^{-\frac{x^{2}}{2}}-\left(3 x+x^{3}\right) \int_{x}^{\infty} e^{-\frac{r^{2}}{2}} d r\right], & x \geq \bar{c}_{1}, \\ 3 \underline{\sigma}^{2} x+x^{3}+\frac{d_{1}}{2 \underline{\sigma^{2}}}\left[\left(2 \underline{\sigma}^{2}+x^{2}\right) e^{-\frac{x^{2}}{2 \underline{\sigma}^{2}}}+\frac{1}{\underline{\sigma}}\left(3 \underline{\sigma}^{2} x+x^{3}\right) \int_{-\infty}^{\frac{\underline{x}}{\underline{\sigma}}} e^{-\frac{r^{2}}{2}} d r\right], & x<\bar{c}_{1},\end{cases}
$$

with $\bar{c}_{1}=-0.5840, k_{1}=0.6154$ and $d_{1}=36.8406$. The exact solution at $\left(t_{0}, x_{0}\right)$ is $\left(u(0,0), \partial_{x} u(0,0)\right)=\left(Y_{0}, Z_{0}\right)=(-0.2595,1.3331)$. We solve this example by Scheme 2 and Scheme 3 with $Q_{z}^{n}=Q_{y}^{n}$. The numerical errors and the corresponding convergence rates are listed in Table 1, which are consistent with our theoretical results.

Next, we choose a different $c_{1}=0$. The unique solution of the corresponding $G$-heat equation is $u(t, x)=(1-t)^{\frac{3}{2}} P\left(\frac{x}{\sqrt{1-t}}\right)$, and the exact solution is $\left(u(0,0), \partial_{x} u(0,0)\right)=\left(Y_{0}, Z_{0}\right)=$ $(0.6154,1.8430)$. We solve this example by Scheme 2 and Scheme 3 with $Q_{z}^{n}=Q_{y}^{n}$ again, and the errors and the corresponding convergence rates are given in Table 2. 
Table 1: Errors and convergence rates for Example 6.1 with $c_{1}=-0.584$.

\begin{tabular}{||c|c|c|c|c|c|c||}
\hline \multicolumn{7}{||c|}{$\mid Y_{0}-Y^{0}$} \\
\hline Scheme & $N=16$ & $N=32$ & $N=64$ & $N=128$ & $N=256$ & CR \\
\hline TR & $9.347 \mathrm{E}-04$ & $6.110 \mathrm{E}-04$ & $4.286 \mathrm{E}-04$ & $2.886 \mathrm{E}-04$ & $1.764 \mathrm{E}-04$ & 0.589 \\
\hline GH & $7.005 \mathrm{E}-03$ & $4.966 \mathrm{E}-03$ & $3.259 \mathrm{E}-03$ & $2.002 \mathrm{E}-03$ & $1.158 \mathrm{E}-03$ & 0.650 \\
\hline \multicolumn{7}{||c|}{$\mid Z_{0}-Z^{0}$} \\
\hline Scheme & $N=16$ & $N=32$ & $N=64$ & $N=128$ & $N=256$ & CR \\
\hline TR & $1.216 \mathrm{E}-01$ & $7.390 \mathrm{E}-02$ & $4.183 \mathrm{E}-02$ & $2.203 \mathrm{E}-02$ & $1.088 \mathrm{E}-02$ & 0.871 \\
\hline GH & $4.918 \mathrm{E}-02$ & $3.377 \mathrm{E}-02$ & $2.320 \mathrm{E}-02$ & $1.520 \mathrm{E}-02$ & $9.348 \mathrm{E}-03$ & 0.594 \\
\hline
\end{tabular}

Table 2: Errors and convergence rates for Example 6.1 with $c_{1}=0$.

\begin{tabular}{||c|c|c|c|c|c|c||}
\hline \multicolumn{7}{||c|}{$\mid Y_{0}-Y^{0}$} \\
\hline Scheme & $N=16$ & $N=32$ & $N=64$ & $N=128$ & $N=256$ & CR \\
\hline TR & $9.097 \mathrm{E}-03$ & $4.294 \mathrm{E}-03$ & $2.564 \mathrm{E}-03$ & $1.123 \mathrm{E}-03$ & $4.811 \mathrm{E}-04$ & 1.042 \\
\hline GH & $1.455 \mathrm{E}-02$ & $7.413 \mathrm{E}-03$ & $3.965 \mathrm{E}-03$ & $1.900 \mathrm{E}-03$ & $9.534 \mathrm{E}-04$ & 0.983 \\
\hline \multicolumn{7}{||c|}{$\mid Z_{0}-Z^{0}$} \\
\hline Scheme & $N=16$ & $N=32$ & $N=64$ & $N=128$ & $N=256$ & CR \\
\hline TR & $7.694 \mathrm{E}-02$ & $3.975 \mathrm{E}-02$ & $1.843 \mathrm{E}-02$ & $9.404 \mathrm{E}-03$ & $5.028 \mathrm{E}-03$ & 0.995 \\
\hline GH & $1.328 \mathrm{E}-02$ & $6.853 \mathrm{E}-03$ & $5.800 \mathrm{E}-03$ & $2.434 \mathrm{E}-03$ & $1.072 \mathrm{E}-03$ & 0.876 \\
\hline
\end{tabular}

By contrast, we observed that when $c_{1}=0$, the convergence rates of $\left|Y_{0}-Y^{0}\right|$ and $\left|Z_{0}-Z^{0}\right|$ are higher than that when $c_{1}=-0.5840$. This is due to the fact that $x_{0}=0$ is the inflection point of the solution $u(0, x)$ in the case when $c_{1}=-0.5840$, but $x_{0}=0$ is not the inflection point of $u(0, x)$ when $c_{1}=0$. At the convex or concave point, the calculation of G-Brownian motion distribution degenerates to that of classical Brownian motion distribution, which leads to a higher convergence rate than the theoretical result. However, the conclusion does not hold at the inflection point.

\section{2. $G-F B S D E s$}

In the following, we will apply our schemes to solve $G$-FBSDEs. The first example is the case of one-dimensional $G$-Brownian motion, and the second is the multi-dimensional case.

\section{Example 6.2. We consider the following G-FBSDEs:}

$$
\left\{\begin{aligned}
X_{t} & =x_{0}+\int_{0}^{t} \frac{1}{1+2 \exp \left(s+X_{s}\right)} d s+\int_{0}^{t} \frac{\exp \left(s+X_{s}\right)}{1+\exp \left(s+X_{s}\right)} d B_{s}, \quad 0 \leq t \leq T, \\
Y_{t} & =\frac{\exp \left(T+X_{T}\right)}{1+\exp \left(T+X_{T}\right)}-\int_{t}^{T}\left[\frac{Y_{s}}{1+2 \exp \left(s+X_{s}\right)}+G\left(\varphi\left(2 Y_{s}^{2}\right)-1\right)\right] d s \\
& -\frac{1}{2} \int_{t}^{T}\left[1+\frac{\varphi\left(Y_{s}\right) \varphi\left(Z_{s}\right)}{1+\exp \left(s+X_{s}\right)}-\varphi\left(Y_{s}^{2}\right)\left(2+\varphi\left(Z_{s}\right)\right)\right] d\langle B\rangle_{s}-\int_{t}^{T} Z_{s} d B_{s}-\left(K_{T}-K_{t}\right) .
\end{aligned}\right.
$$


The analytic solution of $(6.1)$ is

$$
\left\{\begin{array}{l}
Y_{t}=\frac{\exp \left(t+X_{t}\right)}{1+\exp \left(t+X_{t}\right)}, \quad Z_{t}=\frac{\left(\exp \left(t+X_{t}\right)\right)^{2}}{\left(1+\exp \left(t+X_{t}\right)\right)^{3}} \\
K_{t}=\int_{0}^{t}\left[\frac{\left(\exp \left(s+X_{s}\right)\right)^{2}}{\left(1+\exp \left(s+X_{s}\right)\right)^{2}}-\frac{1}{2}\right] d\langle B\rangle_{s}-\int_{0}^{t} G\left(\frac{2\left(\exp \left(s+X_{s}\right)\right)^{2}}{\left(1+\exp \left(s+X_{s}\right)\right)^{2}}-1\right) d s .
\end{array}\right.
$$

Set $T=1, x_{0}=1, \underline{\sigma}=0.7$, and $\bar{\sigma}=1$. The exact solution is $\left(Y_{0}, Z_{0}\right)=(0.731,0.144)$. We test this example by Scheme 2 and Scheme 3 with different $Q_{z}^{n}$. The numerical errors and the corresponding convergence rates for $N=16,32,64,128,256$ are listed in Table 3. It is clear that the selection of $Q_{z}^{n}$ in Scheme 3 does not affect the convergence rate, which is consistent with our theoretical results.

Table 3: Errors and convergence rates for Example 6.2.

\begin{tabular}{|c|c|c|c|c|c|c|c|}
\hline \multicolumn{8}{|c|}{$\left|Y_{0}-Y^{0}\right|$} \\
\hline \multicolumn{2}{|c|}{ Scheme } & $N=16$ & $N=32$ & $N=64$ & $N=128$ & $N=256$ & $\mathrm{CR}$ \\
\hline \multicolumn{2}{|r|}{ TR } & $1.656 \mathrm{E}-03$ & 7.642E-04 & $3.746 \mathrm{E}-04$ & $1.808 \mathrm{E}-04$ & $8.960 \mathrm{E}-05$ & 1.050 \\
\hline \multirow{3}{*}{$\mathrm{GH}$} & $Q_{z}^{n}=Q_{y}^{n}$ & $1.890 \mathrm{E}-03$ & 8.803E-04 & $4.246 \mathrm{E}-04$ & $2.056 \mathrm{E}-04$ & $1.016 \mathrm{E}-04$ & 1.053 \\
\hline & $Q_{z}^{n}=\underline{\sigma}$ & $1.832 \mathrm{E}-03$ & $8.519 \mathrm{E}-04$ & $4.105 \mathrm{E}-04$ & $1.985 \mathrm{E}-04$ & $9.806 \mathrm{E}-04$ & 1.055 \\
\hline & $Q_{z}^{n}=\bar{\sigma}$ & $1.888 \mathrm{E}-03$ & $8.796 \mathrm{E}-04$ & $4.243 \mathrm{E}-04$ & $2.054 \mathrm{E}-04$ & $1.015 \mathrm{E}-04$ & 1.053 \\
\hline \multicolumn{8}{|c|}{$Z_{0}-Z^{0}$} \\
\hline \multicolumn{2}{|c|}{$\begin{array}{c}\text { Scheme } \\
\text { TR }\end{array}$} & $N=16$ & $N=32$ & $N=64$ & $N=128$ & $N=256$ & $\mathrm{CR}$ \\
\hline \multicolumn{2}{|r|}{ TR } & $5.004 \mathrm{E}-03$ & $2.152 \mathrm{E}-03$ & $9.975 \mathrm{E}-04$ & $4.908 \mathrm{E}-04$ & $2.657 \mathrm{E}-04$ & 1.060 \\
\hline & $Q_{z}^{n}=Q_{y}^{n}$ & $5.731 \mathrm{E}-03$ & $2.625 \mathrm{E}-03$ & $1.213 \mathrm{E}-03$ & $5.666 \mathrm{E}-04$ & $2.745 \mathrm{E}-04$ & 1.098 \\
\hline \multirow[t]{2}{*}{$\mathrm{GH}$} & $Q_{z}^{n}=\underline{\sigma}$ & $5.381 \mathrm{E}-03$ & $2.398 \mathrm{E}-03$ & $1.105 \mathrm{E}-03$ & $5.308 \mathrm{E}-04$ & $2.731 \mathrm{E}-04$ & 1.078 \\
\hline & $Q_{z}^{n}=\bar{\sigma}$ & $5.728 \mathrm{E}-03$ & $2.624 \mathrm{E}-03$ & $1.212 \mathrm{E}-03$ & $5.661 \mathrm{E}-04$ & $2.743 \mathrm{E}-04$ & 1.098 \\
\hline
\end{tabular}

Example 6.3. In this example, let $G(\cdot)$ be a sublinear function defined in (5.1) with $\Sigma=$ $\left\{\lambda Q_{1}+(1-\lambda) Q_{2}, \lambda \in[0,1]\right\}$, where

$$
Q_{1}=\left(\begin{array}{ll}
2 & 1 \\
1 & 1
\end{array}\right), \quad Q_{2}=\left(\begin{array}{ll}
1 & 1 \\
1 & 2
\end{array}\right) .
$$

Let $B_{t}=\left(B_{t}^{1}, B_{t}^{2}\right)^{\top}$ be a two-dimensional $G$-Brownian motion. We apply Scheme 3 to the following $G$-FBSDEs:

$$
\left\{\begin{array}{l}
Y_{t}=Y_{T}-\int_{t}^{T}\left(Z_{s}^{1}+Z_{s}^{2}+G\left(M_{s}\right)\right) d s-\int_{t}^{T} Z_{s} d B_{s}-\left(K_{T}-K_{t}\right), \\
Y_{T}=\sin \left(T+B_{T}^{1}\right) \cos \left(T+B_{T}^{2}\right)
\end{array}\right.
$$

where $Z_{s}=\left(Z_{s}^{1}, Z_{s}^{2}\right), M_{s}=\left(\begin{array}{cc}-Y_{s} & V_{s} \\ V_{s} & -Y_{s}\end{array}\right)$, and $V_{s}=-\cos \left(s+B_{s}^{1}\right) \sin \left(s+B_{s}^{2}\right)$. The analytic solution of $(6.2)$ is given by

$$
\left\{\begin{array}{l}
Y_{t}=\sin \left(t+B_{t}^{1}\right) \cos \left(t+B_{t}^{2}\right), \\
Z_{t}=\left(\cos \left(t+B_{t}^{1}\right) \cos \left(t+B_{t}^{2}\right),-\sin \left(t+B_{t}^{1}\right) \sin \left(t+B_{t}^{2}\right)\right), \\
K_{t}=\frac{1}{2} \int_{0}^{t} M_{s} d\langle B\rangle_{s}-\int_{0}^{t} G\left(M_{s}\right) d s .
\end{array}\right.
$$


Set $T=1, x_{0}=1$, and $L=6$. The exact solution is $\left(Y_{0}, Z_{0}\right)=(0,(1,0))$. In our tests, we found that the difference between choosing different $Q_{z}^{n} \in\left\{Q_{1}, Q_{2}\right\}$ is negligible. We only list the numerical errors and the convergence rates for $N=16,32,64,128,256$ with $Q_{z}^{n}=Q_{1}$ in Table 4. It is shown that our method is stable and has high accuracy.

Table 4: Errors and convergence rates of Scheme 3 for Example 6.3.

\begin{tabular}{|c|c|c|c|c|c|c|}
\hline & & & \multicolumn{4}{|l|}{$Y_{0}-Y^{0}$} \\
\hline GH & $N=16$ & $N=32$ & $N=64$ & $N=128$ & $N=256$ & $\mathrm{CR}$ \\
\hline$Q_{z}^{n}=Q_{1}$ & $1.838 \mathrm{E}-01$ & $9.928 \mathrm{E}-02$ & $5.015 \mathrm{E}-02$ & $2.497 \mathrm{E}-02$ & $1.243 \mathrm{E}-02$ & 0.976 \\
\hline & & & \multicolumn{4}{|l|}{$Z_{0}-Z^{0}$} \\
\hline GH & $N=16$ & $N=32$ & $N=64$ & $N=128$ & $N=256$ & CR \\
\hline$Q_{z}^{n}=Q_{1}$ & $1.631 \mathrm{E}-01$ & $5.685 \mathrm{E}-02$ & $1.980 \mathrm{E}-02$ & $7.609 \mathrm{E}-03$ & $3.251 \mathrm{E}-03$ & 1.420 \\
\hline
\end{tabular}

\section{Conclusions}

In this paper, we propose some efficient numerical schemes for solving G-FBSDEs. With the help of the $G$-expectation representation, we design a feasible method to approximate quadrature rule to approximate the distribution of $G$-Brownian motion, we propose some new numerical schemes for solving $G$-FBSDEs. We also rigorously analyze the errors of our proposed schemes and prove the convergence results. Several numerical examples are presented to show the effectiveness of our numerical schemes.

\section{References}

[1] M. Avellaneda, A. Levy, A. Paras, Pricing and hedging derivative securities in markets with uncertain volatilities, Appl. Math. Financ. 2(1995) 73-88.

[2] V. Bally, Approximation scheme for solutions of BSDE, in: N.E. Karoui, L. Mazliak (Eds.), Backward Stochastic Differential Equations, Addison Wesley Longman, Harlow, UK, 1997, pp. 177-191.

[3] C. Bender, R. Denk, A forward scheme for backward SDEs, Stochastic Process. Appl. 117 (2007) 1793-1812.

[4] J.F. Chassagneux, Linear Multistep Schemes for BSDEs, SIAM J. Numer. Anal. 52 (2014) 2815-2836. 
[5] J.F. Chassagneux, A. Richou, Numerical stability analysis of the Euler scheme for BSDEs, SIAM J. Numer. Anal. 53 (2015) 1172-1193.

[6] P. Cheridito, H.M. Soner, N. Touzi, N. Victoir, Second-order backward stochastic differential equations and fully nonlinear parabolic PDEs, Comm. Pure Appl. Math. 60 (2007) 1081-1110.

[7] D. Crisan, K. Manolarakis, Second order discretization of a backward SDEs and simulation with the cubature method, Ann. Appl. Probab. 24 (2014) 652-678.

[8] C. Dellacherie, Capacités et Processus Stochastiques, Springer Verlag, Berlin, 1972.

[9] L. Denis, C. Martini, A theorical framework for the pricing of continent claims in the presence of model uncertainty, Ann. Appl. Probab. 16 (2006) 827-852.

[10] L. Denis, M. Hu, S. Peng, Function spaces and capacity related to a sublinear expectation: application to G-Brownian motion paths, Potential Anal. 34 (2011) 139-161.

[11] Y. Dolinsky, Numerical schemes for G-expectations, Electron. J. Probab. 17 (2012) 1-15.

[12] W. E, J. Han, A. Jentzen, Deep Learning-Based Numerical Methods for HighDimensional Parabolic Partial Differential Equations and Backward Stochastic Differential Equations, Commun. Math. Stat. 5 (2017) 349-380.

[13] A. Fahim, N. Touzi, X. Warin, A probabilistic numerical method for fully nonlinear parabolic PDEs, Ann. Appl. Probab. 21 (2011) 1322-1364.

[14] E. Gobet, J.-P. Lemor, X. Warin, A regression-based Monte Carlo method for backward stochastic differential equations, Ann. Appl. Probab. 15 (2005) 2172-2202.

[15] E. Gobet, P. Turkedjiev, Linear regression MDP scheme for discrete backward stochastic differential equations under general conditions, Math. Comput. 85 (2016) 1359-1391.

[16] W. Guo, J. Zhang, J. Zhuo, A monotone scheme for high-dimensional fully nonlinear PDEs, Ann. Appl. Probab. 25 (2015) 1540-1580.

[17] M. Hu, S. Peng, On representation theorem of $G$-expectations and paths of $G$-Brownian motion, Acta Math. Appl. Sin. Engl. Ser. 25 (2009) 539-546. 
[18] M. Hu, Explicit solutions of $G$-heat equation with a class of initial conditions by $G$ Brownian motion, Nonlinear Anal. 75 (2012) 6588-6595.

[19] M. Hu, S. Ji, S. Peng, Y. Song, Backward stochastic differential equations driven by G-Brownian motion, Stoch. Proc. Appl. 124 (2014) 759-784.

[20] M. Hu, S. Ji, S. Peng, Y. Song, Comparison theorem, Feynman-Kac formula and Girsanov transformation for BSDEs driven by $G$-Brownian motion, Stoch. Proc. Appl. 124 (2014) 1170-1195.

[21] T. Kong, W. Zhao, T. Zhou, Probabilistic high order numerical schemes for fully nonlinear parabolic PDEs, Commun. Comput. Phys. 18 (2015) 1482-1503.

[22] N.V. Krylov, Nonlinear Parabolic and Elliptic Equations of the Second Order, Reidel Publishing Company, 1987 (Original Russian Version by Nauka, Moscow, 1985).

[23] T.J. Lyons, Uncertain volatility and the risk-free synthesis of derivatives, J. Appl. Finance 2 (1995) 117-133.

[24] J. Ma, J. Zhang, Representations and regularities for solutions to BSDEs with reflections, Stochastic Process. Appl. 115 (2005) 539-569.

[25] G.N. Milstein, M.V. Tretyakov, Numerical algorithms for forward-backward stochastic differential equations, SIAM J. Sci. Comput. 28 (2006) 561-582.

[26] S. Peng, Filtration consistent nonlinear expectations and evaluations of contingent claims, Acta Math. Appl. Sin. 20 (2004) 1-24.

[27] S. Peng, G-expectation, G-Brownian motion and related stochastic calculus of Itô type, in: Stochastic Analysis and Applications, in: Abel Symp., vol. 2, Springer, Berlin, 2007, pp. 541-567.

[28] S. Peng, Multi-dimensional G-Brownian motion and related stochastic calculus under G-expectation, Stochastic Process. Appl. 118 (2008) 2223-2253.

[29] S. Peng, Nonlinear Expectations and Stochastic Calculus under Uncertainty-with Robust CLT and G-Brownian Motion, Springer, 2019. 
[30] M.J. Ruijter, C.W. Oosterlee, Numerical Fourier method and second-order Taylor scheme for backward sdes in finance, Appl. Numer. Math. 103 (2016) 1-26.

[31] M. Soner, N. Touzi, J. Zhang, Wellposedness of second order backward SDEs, Probab. Theory Relat. Fields 153 (2012) 149-190.

[32] L. Teng, A. Lapitckii, M. Gunther, A multi-step scheme based on cubic spline for solving backward stochastic differential equations, Appl. Numer. Math. 150 (2020) 117-138.

[33] J. Yang, W. Zhao, Numerical simulations for G-Brownian motion, Front. Math. China 6 (2016) 1625-1643.

[34] J. Zhang, A numerical scheme for BSDEs, Ann. Appl. Probab. 14 (2004) 459-488.

[35] Y. Zhang, W. Zheng, Discretizing a backward stochastic differential equation, Int. J. Math. Math. Sci. 32 (2002) 103-116.

[36] W. Zhao, L. Chen, S. Peng, A new kind of accurate numerical method for backward stochastic differential equations, SIAM J. Sci. Comput. 28 (2006) 1563-1581.

[37] W. Zhao, Y. Fu, T. Zhou, New kinds of high-order multistep schemes for coupled forward backward stochastic differential equations, SIAM J. Sci. Comput. 36 (2014) A1731-A1751. 